\title{
Performance Improvement of Membrane Stress Measurement Equipment through Evaluation of Added Mass of Membrane and Error Correction
}

\author{
Sang-Wook Jin, ${ }^{1}$ Hiroshi Ohmori, ${ }^{2}$ and Seungjae Lee ${ }^{1}$ \\ ${ }^{1}$ Department of Architectural Engineering, Korea University of Technology and Education, Cheonan 31253, Republic of Korea \\ ${ }^{2}$ Graduate School of Environmental Studies, Nagoya University, Furo-cho, Chikusa-ku, Nagoya 464-8601, Japan \\ Correspondence should be addressed to Seungjae Lee; leeseung@koreatech.ac.kr
}

Received 28 February 2017; Accepted 27 June 2017; Published 9 August 2017

Academic Editor: Tony Murmu

Copyright (C) 2017 Sang-Wook Jin et al. This is an open access article distributed under the Creative Commons Attribution License, which permits unrestricted use, distribution, and reproduction in any medium, provided the original work is properly cited.

\begin{abstract}
One of the most important issues in keeping membrane structures in stable condition is to maintain the proper stress distribution over the membrane. However, it is difficult to determine the quantitative real stress level in the membrane after the completion of the structure. The stress relaxation phenomenon of the membrane and the fluttering effect due to strong wind or ponding caused by precipitation may cause severe damage to the membrane structure itself. Therefore, it is very important to know the magnitude of the existing stress in membrane structures for their maintenance. The authors have proposed a new method for separately estimating the membrane stress in two different directions using sound waves instead of directly measuring the membrane stress. The new method utilizes the resonance phenomenon of the membrane, which is induced by sound excitations given through an audio speaker. During such experiment, the effect of the surrounding air on the vibrating membrane cannot be overlooked in order to assure high measurement precision. In this paper, an evaluation scheme for the added mass of membrane with the effect of air on the vibrating membrane and the correction of measurement error is discussed. In addition, three types of membrane materials are used in the experiment in order to verify the expandability and accuracy of the membrane measurement equipment.
\end{abstract}

\section{Introduction}

Membrane structure is a system that is stabilized by maintaining a tensile state of the membrane material, which originally cannot resist bending or pressure. Also, it is a system that allows the whole membrane structure to bear external loads caused by wind or precipitations such as rain and snow and other design loads. Due to the innate characteristic of the membrane material, stress relaxation phenomenon can transpire to the stress that is introduced to the fabric over time. Such factor is considered during design; however, lack of consistent maintenance can lead to further rupture of the membrane through the fluttering and ponding phenomena. Thus, it is important to accurately understand the size of the membrane stress after the completion of the structure for maintenance and management purposes.

Until recently, several methods for the measurement of the membrane stress have been proposed, and some have been used in construction sites as trials to obtain precise measurements of the membrane stress. However, the accuracy and stability of the obtained data have been found to be far from the desired outcome. This situation has not changed until very recently, and there has yet been a reliable measurement tool. Moreover, due to different properties of biaxial directions in the membrane materials, the stress in the warp direction is generally different from that in the fill one. Such difference in stress due to directional separation is another factor that causes difficulty measuring the stress of membrane structures.

There are not many techniques for the measurement of the membrane stress. However, the existing techniques can be divided into two methods based on the different usage between displacement and vibration of the membrane in their methods. Sasaki et al. proposed measurement methods to estimate the measurement of membrane displacement when suction is applied by air or when the membrane material 


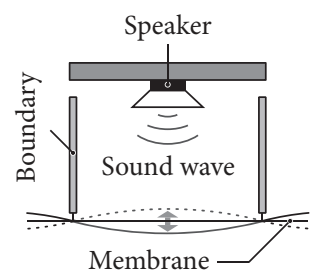

FIgURE 1: Equipment diagram.

is pulled [1, 2]. Yuzuba et al. also developed apparatus based on membrane reaction force of road cell when the membrane surface is pressed [3]. Recently, there also has been a research by Jung et al. who developed the stress measurement equipment that employs membrane stress measurement method utilizing membrane displacement. This equipment contains the most suitable shape and size of the pressing metal part, which was discovered through experiments that applies various shapes and sizes to the metal that puts pressure onto the membrane [4].

On the other hand, Tanizawa developed a measurement method that employs membrane vibration. This method is used to estimate membrane stress and measures the amplitude of the membrane that are vibrated by an impact force, such as a hammer [5]. Also, Sasaki developed a method that allows the measurement of the membrane stress through comparing the sound data of the setup membrane stress that has been already gathered in the laboratory, to the sound that has been gathered and evaluated when the hammer resonates the membrane in the actual site [6].

Some of these methods and equipment have been used in actual construction sites. However, the existing measurement methods generally base their measurements on empirical methods of corresponding the pregathered displacement or resonance data that occurs in the laboratory under the setup stress, to the displacement or resonance data measured in the site structures. For such reasons, there is no practical device, by which membrane stress in both directions can be precisely measured. The measurement of membrane strength at the construction site has solely depended on the experience and knowledge of technical membrane experts until now. This is why new methods to correctly measure membrane stress in both directions are highly anticipated.

The authors have proposed a new estimation method that can evaluate the membrane stress in two different directions using sound waves instead of measuring the membrane stress directly, as shown in Figure 1, and has conducted experimental tests of the membrane stress estimation that use sound excitation with sine wave and white noise [7]. The new method makes use of the resonance phenomena of the vibrating membrane with a rectangular boundary, as shown in Figure 2, which is induced by sound excitation given through an audio speaker. For the current research, the authors have taken into account that the effect of the surrounding air in a vibrating membrane should be considered, due to the fact that the membrane is too light to be unaffected by air while vibrating, and also because it is necessary to consider the added mass of the membrane with the effect of

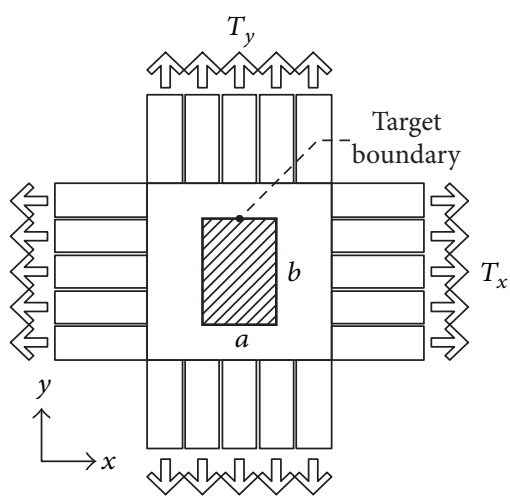

Figure 2: Boundary of the material.

air to theoretically determine the resonance frequency of the membrane with high precision.

In this paper, factors for improving the accuracy of the equipment and experimental tests to verify the improved accuracy and expandability of the measurement equipment with white noise sound excitation and the setting of three membrane material types are presented. In addition, not only the theoretical evaluation scheme of the added mass of the membrane on the effect of air with the vibrating membrane but also the correction of errors for improving estimation accuracy is discussed.

\section{Theory for Estimating Membrane Stress}

In the previous research [7], by assuming that there are only existing tensions of $T_{x}$ and $T_{y}$ per length in $x$ and $y$ directions on the membrane material and due to nonexistence of shear stiffness in the membrane, prestress $T_{x y}$ is zero, which is woven by threads in two orthogonal directions. Existing stresses of $T_{x}$ and $T_{y}$ on the membrane material are obtained by the following equation:

$$
\{T\}=[A]^{-1}\{f\},
$$

where $\{T\}$, $[A]$, and $\{f\}$ are presented as follows:

$$
\begin{aligned}
& \{T\}=\left\{\begin{array}{l}
T_{x} \\
T_{y}
\end{array}\right\}, \\
& {[A]=\left[\begin{array}{ll}
\frac{m_{1}^{2}}{a_{1}^{2}} & \frac{n_{1}^{2}}{b_{1}^{2}} \\
\frac{m_{2}^{2}}{a_{2}^{2}} & \frac{n_{2}^{2}}{b_{2}^{2}}
\end{array}\right],} \\
& \{f\}=4 \rho_{k}\left\{\begin{array}{l}
f_{1}^{2} \\
f_{2}^{2}
\end{array}\right\},
\end{aligned}
$$

where $m_{1,2}$ and $n_{1,2}$ represent the number of waves in the vibration mode, $a_{1}, b_{1}$ and $a_{2}, b_{2}$ represent the side lengths of the rectangular boundary when the external excitation with the respective frequencies $f_{1}$ and $f_{2}$ is observed, and $\rho_{k}$ is the membrane mass per unit area, respectively. 


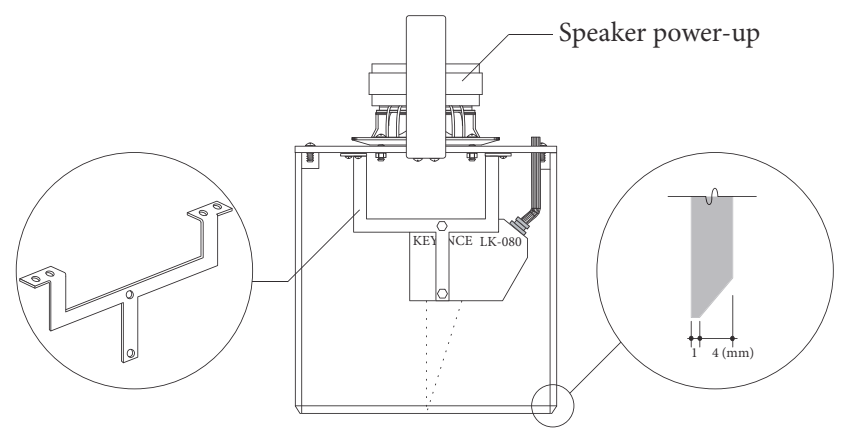

FIGURE 3: Improvement of the measurement equipment.

From (1), if the natural mode of vibration and natural frequency are measured in the resonance when the membrane with the boundary is at the state of adequate vibration, it is possible to separately obtain the objective values of stress, $T_{x}$ and $T_{y}$, within the membrane in both the warp and fill directions. The validity of this new theory for estimating membrane stress has been verified through the experimental tests with sine wave sweep and white noise wave external excitation [7].

\section{Experimental Tests}

3.1. Improvement of the Equipment. Although sine wave sound is generally used to apply force to any object, it has been clearly shown that the white noise sound source is more useful for external excitation, because the sine wave sweep requires a long radiation time and has the possibility of producing response amplitude depending on the speed or time of the sweep. Factors including this sound source that can improve the accuracy of the measurement equipment or reduce the measurement time are considered.

As shown in Figure 3, in order to make the vibration boundary of the membrane material more accurate, the bottom of the acrylic box contacting the membrane surface is of $1 \mathrm{~mm}$ thick sharp edges, unlike $5 \mathrm{~mm}$ thick flat edges of the previous equipment. Also, the total weight of the equipment is increased approximately $500 \mathrm{~g}$ from the previous $3.3 \mathrm{~kg}$, and such increase is expected to have an effect equivalent to the added weight. In addition, the metal connector for fastening the laser displacement sensor to the equipment is designed to be strong enough to withstand the vibration of the sound. Also, the speaker is replaced with a new one, which now allows the power of the speaker to be five times stronger than the 50 watts of the previous speaker. This replacement is to ensure sufficient vibration amplitude, especially when high stress is introduced in the membrane.

3.2. Equipment for Experimental Tests. Experimental tests are carried out to verify the accuracy of the improved equipment mentioned above and also to verify the expandability of such equipment on all types of membrane structures in the construction sites. As shown in Figure 4, the equipment used for the experimental tests is composed of three parts. First, the measurement components consist of an improved
TABLE 1: Capacity of laser displacement sensor [8].

\begin{tabular}{lccc}
\hline Manufacturer Model number & $\begin{array}{c}\text { Resolution } \\
\text { capability }\end{array}$ & Frequency range \\
\hline Keyence Corp. & LK-500 & $10 \mu \mathrm{m}$ & $1024 \mu \mathrm{s}$ \\
\hline
\end{tabular}

audio speaker for sound excitation, a laser displacement for measuring displacement of the vibrating membrane, and an acrylic box for making a fixed boundary on the membrane. Next, the sound source components consist of a computer generating a sound source such as white noise and an amplifier amplifying the sound source. Lastly, the recording and analysis components consist of a data recorder for recording and analyzing the data from the laser displacement.

Figure 5 shows the completed system of measurement components with a total weight of $3.8 \mathrm{~kg}$. By setting the equipment gently on the membrane surface, a small rectangular area is made on the membrane. The transparent acrylic box measures $200 \mathrm{~mm} \times 300 \mathrm{~mm}$ on its sides and $200 \mathrm{~mm}$ in height. The top of the acrylic box of the measurement equipment is left open in order to allow free air movement in and out of the box during vibration. Sound is emanated from the speaker with a white noise wave, which has a defined strength and frequency on the tests, and the laser displacement sensor, which has a capacity as shown in Table 1 [8], measures the displacement of the membrane that resonates by the sound wave without contact. As shown in Figure 5, the bottom of the acrylic box contacting the membrane surface has sharp edges of $1 \mathrm{~mm}$ thickness, so as to make a clear boundary division on the membrane. Figure 6 shows the sound source and the recording and analysis components used in the experimental tests.

3.3. White Noise Wave External Excitation. Even though the sine wave sound is generally used to apply force to an object without contacting it, the white noise wave sound source, which has a 0 to $500 \mathrm{~Hz}$ frequency field for external excitation, is used to vibrate the membrane in these experimental tests. The white noise sound wave is used instead of the sine wave sound source because the external excitation of the sine wave sweep needs a long radiation time, which might be a greater disadvantage for its practical use [7].

The membrane materials are classified depending on their characteristics, as they have different coatings on the woven cloth as shown in Table 2. The measurement equipment is theoretically applicable to any type of membrane material by changing the area density, $\rho_{k}$, of the membrane in (1). The membrane area density is a data that can be easily acquired from material property.

Membrane structures generally have a slope; thus it is presumed that a measurer will hold the equipment when measuring at the field. Therefore, the size of the equipment must be portable and also should not be restricted by electric wires for power supply. For field application of the suggested estimation method, certain assignments such as the exclusion of influences due to the measurer's hand tremor and the change of domestic power into battery power supply need attention. First of all in this paper, the most general and 
TABLE 2: Types of membrane material

\begin{tabular}{lccc}
\hline Name & & Membrane material & \\
& Textile & Coating & ${\text { Density }\left(\mathrm{kg} \mathrm{m}^{-2}\right)}^{\text {Approx. } 1.3}$ \\
\hline Type A & Glass fiber & Ethylene fluoride & Approx. 0.9 \\
Type B & Glass fiber & Synthetic resins & Approx. 0.8 \\
Type C & Synthetic fiber and so on & Synthetic resins & . \\
\hline
\end{tabular}

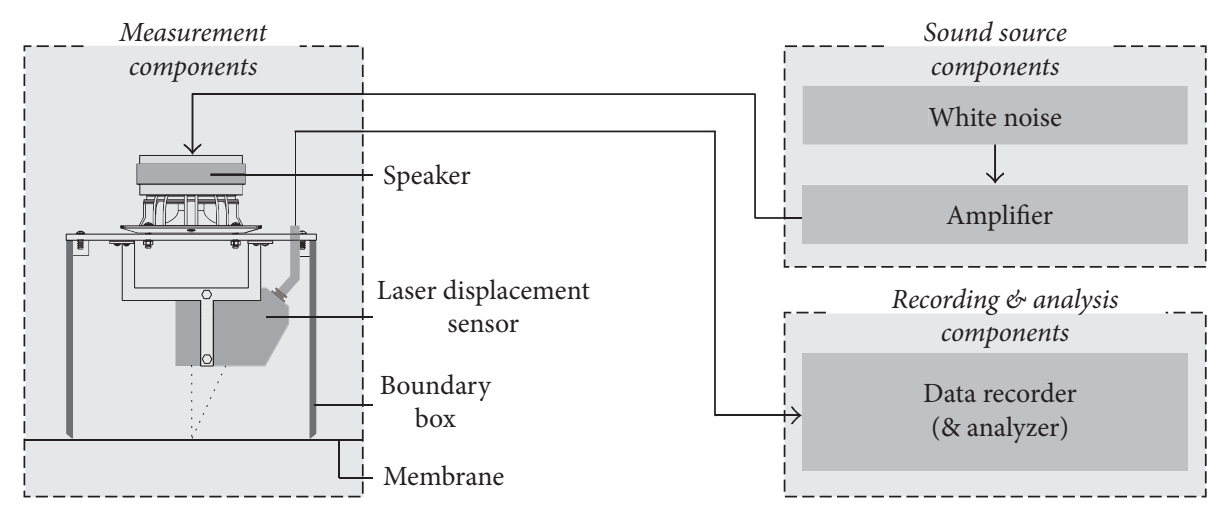

FIGURE 4: Experimental test setup.

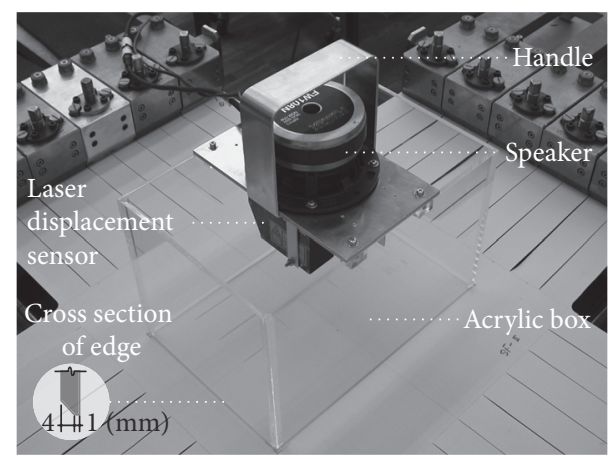

FIGURE 5: Measurement equipment.

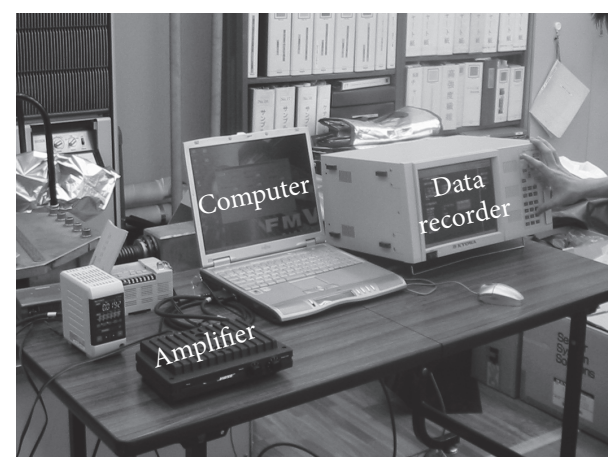

FIGURE 6: Sound source, recording, and analysis components.

typical membrane among the three types of membrane materials $(\mathrm{A}, \mathrm{B}$, and $\mathrm{C})$ is used in the experiment, thus verifying the applicability of the newly suggested theory even in the case of membrane material change.

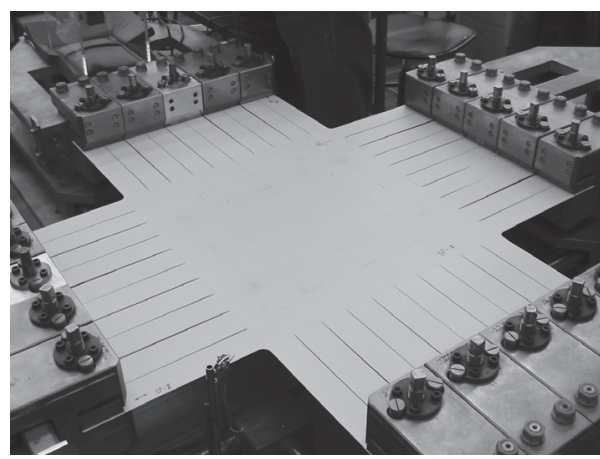

FIGURE 7: Membrane material setup.

3.3.1. Type A Membrane Material. The experimental test using Type A membrane material is carried out by a tensile stress machine, which can introduce stress separately to the membrane in both warp and fill directions. The membrane material used in the experimental test is a type of PTFEcoated glass fiber with a membrane area density of $1.3 \mathrm{~kg} \mathrm{~m}^{-2}$, which is the average value obtained through five times of measurements with that which has the size of $100 \mathrm{~mm} \times$ $100 \mathrm{~mm}$ and the thickness of $0.8 \mathrm{~mm}$. The rough shape of the membrane material is shown in Figures 2 and 7. The material has slits that serve not only to avoid introducing stress vertically in the membrane but also to introduce stress more accurately in the membrane as intended. The membrane material, with the exception of the slits, measures $400 \mathrm{~mm} \times$ $400 \mathrm{~mm}$.

As shown in Table 3, the membrane stress introduced in the experimental test follows three patterns. In one pattern, the interval from 0 to $20 \mathrm{kN} \mathrm{m}^{-1}$ is increased by $1 \mathrm{kN} \mathrm{m}^{-1}$, 
TABLE 3: Patterns of setup stress in white noise excitation (unit: $\mathrm{kN} \mathrm{m}^{-1}$ ).

\begin{tabular}{|c|c|c|c|c|c|c|c|}
\hline \multicolumn{8}{|c|}{ Setup stress } \\
\hline \multicolumn{4}{|c|}{$\begin{array}{c}T_{x}=T_{y}=T_{0} \\
\left(T_{0}: \text { target stress }\right)\end{array}$} & \multicolumn{3}{|c|}{$T_{x} / T_{y}=C_{0}$} & $T_{y} / T_{x}=C_{0}$ \\
\hline$T_{x}$ & $T_{y}$ & $T_{x}$ & $T_{y}$ & $T_{x}$ & $T_{y}$ & $T_{x}$ & $T_{y}$ \\
\hline 1.40 & 0.86 & 10.82 & 10.06 & 1.69 & 1.94 & 2.13 & 1.07 \\
\hline 2.14 & 1.59 & 11.81 & 11.11 & 2.55 & 3.82 & 4.02 & 1.98 \\
\hline 2.94 & 2.36 & 12.67 & 11.99 & 3.44 & 5.57 & 5.65 & 2.81 \\
\hline 4.09 & 3.38 & 14.26 & 13.57 & 4.37 & 7.29 & 7.62 & 3.79 \\
\hline 4.95 & 4.26 & 14.86 & 14.16 & 5.39 & 9.20 & 9.43 & 4.69 \\
\hline 5.86 & 5.13 & 15.53 & 14.87 & 6.33 & 10.95 & 11.49 & 5.76 \\
\hline 6.84 & 6.09 & 16.98 & 16.35 & 7.25 & 12.94 & 13.45 & 6.75 \\
\hline 7.75 & 7.01 & 18.24 & 17.62 & 8.17 & 14.75 & 15.48 & 7.75 \\
\hline 8.75 & 7.96 & 18.64 & 18.04 & 9.15 & 16.88 & 17.25 & 8.65 \\
\hline 9.78 & 8.98 & 19.69 & 19.07 & 10.09 & 18.56 & 19.37 & 9.70 \\
\hline
\end{tabular}

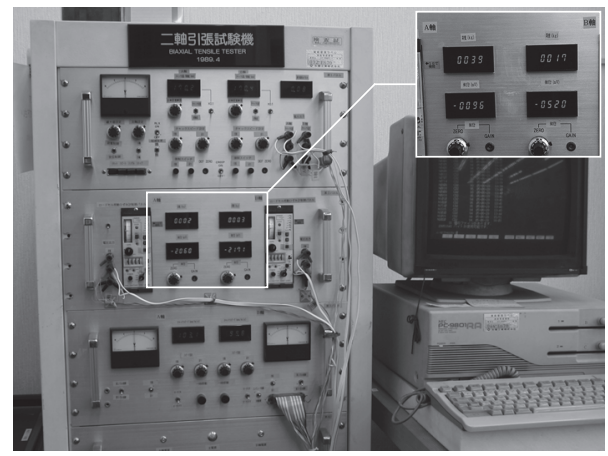

FIGURE 8: Force sensor for introducing stress.

so that the introduced stress ratio will reach $1: 1$ in the $X$ and $Y$ directions. The tensile test is also carried out with the membrane stress ratios of $1: 2$ and $2: 1$ in the $X$ and $Y$ directions. However, as shown in Table 3, there are small differences between the target stress values and the setup stress values.

As shown in Figure 8, the introduced membrane stress is read from the force sensor, which controls the value of the introduced stress. The introduced stress will also be displayed on the force sensor screen in real-time, when the tremor of stress is stabilized after the measurement equipment has been placed on the membrane for a sufficient time. The time for radiating white noise to the membrane is only 10 seconds, making it 50 seconds less than the experimental test used in sine wave external excitation [7]. Figure 9 presents a view of the experimental test where the measurement equipment is gently placed on the membrane material when a specific stress is induced to the membrane.

Measurements are carried out twice in order to record the two different frequencies of each introduced stress, as mentioned in the subsection of the theory for estimating the membrane stress. As for vibration, the first mode $(m=1$, $n=1$ ) is generally prominent in the middle of the designated

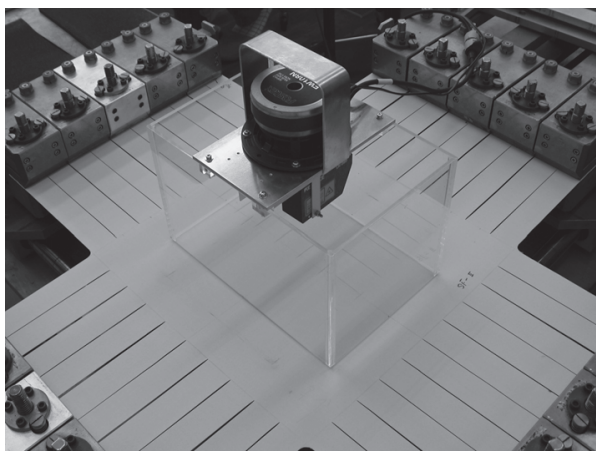

FIgURE 9: Experimental test.

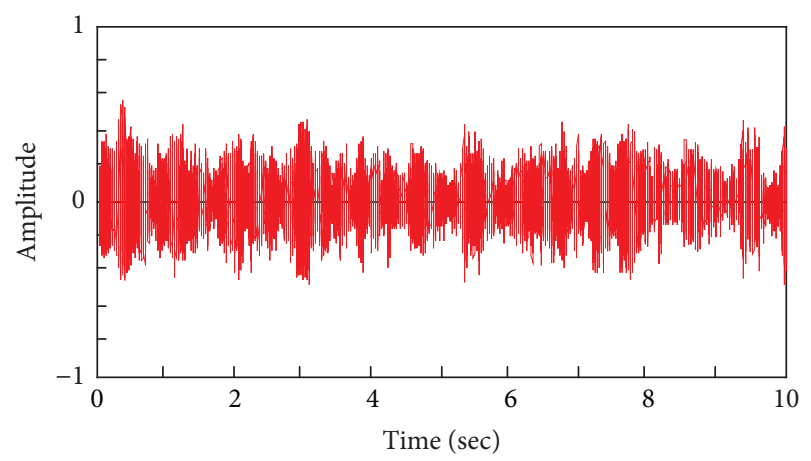

FIgURE 10: Wave data of vibrating membrane.

membrane, in both directions. Thus, $m_{1}, n_{1}, m_{2}$, and $n_{2}$ become " 1 " in (1). It is possible to obtain $f_{1}$, which is the resonance frequency when the boundary lengths are $a_{1}=$ $200 \mathrm{~mm}$ and $b_{1}=300 \mathrm{~mm}$. Then, the boundary box is rotated 90 degrees on the vertical axis, and $f_{2}$ can be obtained when the boundary lengths are $a_{2}=300 \mathrm{~mm}$ and $b_{2}=$ $200 \mathrm{~mm}$.

Figure 10 shows an example of the wave data of the membrane with the given elapsed time, when the membrane 
TABLE 4: Result of the Type A membrane material under setup stress of $T_{x}=T_{y}=T_{0}\left(T_{0}=\right.$ target stress $)$.

\begin{tabular}{|c|c|c|c|c|c|c|c|c|c|c|}
\hline \multirow[t]{2}{*}{ Case } & \multicolumn{2}{|c|}{$\begin{array}{c}T_{x}=T_{y}=T_{0} \\
\left(T_{0}=\text { target stress }\right) \\
\text { Setup stress }\left(\mathrm{kN} \mathrm{m}^{-1}\right)\end{array}$} & \multicolumn{2}{|c|}{$\begin{array}{l}\text { Estimation stress } \\
\qquad\left(\mathrm{kN} \mathrm{m}^{-1}\right)\end{array}$} & \multicolumn{6}{|c|}{ Considering added mass of membrane } \\
\hline & $T_{x}$ & $T_{y}$ & $T_{x}$ & $T_{x}$ & $T_{x}$ & $T_{y}$ & $E_{x}$ & $E_{y}$ & $E_{x}$ & $E_{y}$ \\
\hline 1 & 1.40 & 0.86 & 1.16 & 0.72 & 1.41 & 0.88 & -0.01 & -0.02 & -0.71 & -2.33 \\
\hline 2 & 2.14 & 1.59 & 1.74 & 1.22 & 2.11 & 1.47 & 0.03 & 0.12 & 1.40 & 7.55 \\
\hline 3 & 2.94 & 2.36 & 2.35 & 1.74 & 2.85 & 2.11 & 0.09 & 0.25 & 3.06 & 10.59 \\
\hline 4 & 4.09 & 3.38 & 3.00 & 2.50 & 3.63 & 3.03 & 0.46 & 0.35 & 11.25 & 10.36 \\
\hline 5 & 4.95 & 4.26 & 3.69 & 3.13 & 4.46 & 3.78 & 0.49 & 0.48 & 9.90 & 11.27 \\
\hline 6 & 5.86 & 5.13 & 4.33 & 3.60 & 5.22 & 4.34 & 0.64 & 0.79 & 10.92 & 15.40 \\
\hline 7 & 6.84 & 6.09 & 4.98 & 4.09 & 6.00 & 4.94 & 0.84 & 1.15 & 12.28 & 18.88 \\
\hline 8 & 7.75 & 7.01 & 5.18 & 4.86 & 6.23 & 5.86 & 1.52 & 1.15 & 19.61 & 16.41 \\
\hline 9 & 8.75 & 7.96 & 5.67 & 5.18 & 6.82 & 6.23 & 1.93 & 1.73 & 22.06 & 21.73 \\
\hline 10 & 9.78 & 8.98 & 5.92 & 5.82 & 7.12 & 6.99 & 2.66 & 1.99 & 27.20 & 22.16 \\
\hline 11 & 10.82 & 10.06 & 6.61 & 6.07 & 7.93 & 7.29 & 2.89 & 2.77 & 26.71 & 27.53 \\
\hline 12 & 11.81 & 11.11 & 6.47 & 6.35 & 7.77 & 7.63 & 4.04 & 3.48 & 34.21 & 31.32 \\
\hline 13 & 12.67 & 11.99 & 6.94 & 6.64 & 8.83 & 7.96 & 4.34 & 4.03 & 34.25 & 33.61 \\
\hline 14 & 14.26 & 13.57 & 7.07 & 7.23 & 8.47 & 8.67 & 5.79 & 4.90 & 40.60 & 36.11 \\
\hline 15 & 14.86 & 14.16 & 7.29 & 6.89 & 8.74 & 8.26 & 6.12 & 5.90 & 41.18 & 41.67 \\
\hline 16 & 15.53 & 14.87 & 7.41 & 7.11 & 8.88 & 8.52 & 6.65 & 6.35 & 42.82 & 42.70 \\
\hline 17 & 16.98 & 16.35 & 6.88 & 7.53 & 8.25 & 9.02 & 8.73 & 7.33 & 51.41 & 44.83 \\
\hline 18 & 18.24 & 17.62 & 7.52 & 7.52 & 9.01 & 9.01 & 9.23 & 8.61 & 50.60 & 48.86 \\
\hline 19 & 18.64 & 18.04 & 7.84 & 7.30 & 9.40 & 8.74 & 9.24 & 9.30 & 49.57 & 51.55 \\
\hline 20 & 19.69 & 19.07 & 7.57 & 7.30 & 9.07 & 8.75 & 10.62 & 10.32 & 53.94 & 54.12 \\
\hline Average & & & & & & & 3.82 & 3.55 & 27.11 & 27.22 \\
\hline Standard deviation & & & & & & & 3.48 & 3.28 & 17.64 & 16.03 \\
\hline
\end{tabular}

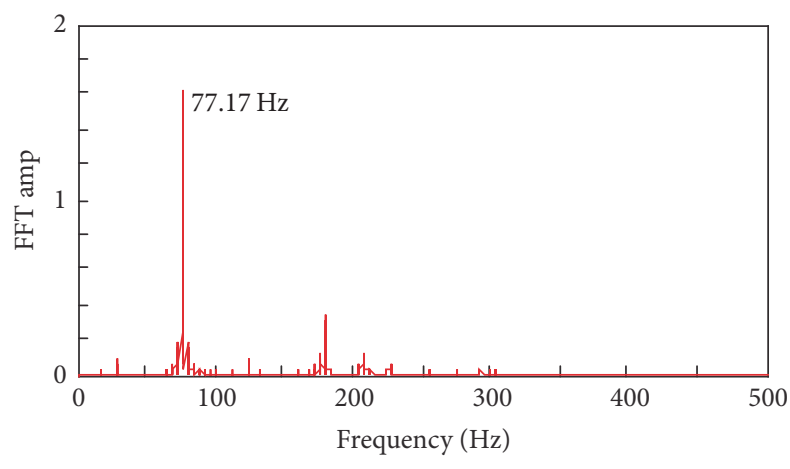

FIGURE 11: Result of FFT analysis of wave data.

vibrates under the specific stress in the experimental test. The horizontal axis indicates time, and the vertical axis indicates the amplitude of the membrane under white noise external excitation. Figure 11 shows the result of fast Fourier transform (FFT) analysis along with Figure 10, where the horizontal axis indicates the amplitude of FFT analysis, while the vertical axis stands for the frequency. As shown in Figure 11, 77.17 Hz is an excellent result compared to other frequencies by
FFT analysis; thus this experiment shows the possibility of determining the resonance frequency.

Tables 4 and 5 show the results of the experiment using white noise for external excitation when the setup stress is, respectively, $T_{x}=T_{y}=T_{0}\left(T_{0}=\right.$ target stress $)$ and $T_{x} / T_{y}$ or $T_{y} / T_{x}=C_{0}\left(C_{0}=\right.$ target ratio $)$. Figure 12 represents the results of Tables 4 and 5 in a graph. The vertical axis indicates the measurement stress obtained when the stress is set as the introduced stress on the horizontal axis. For instance, the symbol "o" around $5 \mathrm{kN} \mathrm{m}^{-1}$ on the horizontal axis shows that the stress of $3.6 \mathrm{kN} \mathrm{m}^{-1}$ is obtained by estimating the $Y$ direction when the $X$ and $Y$ direction target ratio of stress is $T_{x}=T_{y}=T_{0}$. The diagonal solid line in Figure 12 shows the objective accuracy, and the gray zones indicate the margin of accuracy error as 10 to 30 percent. Thus, the discrepancy between the estimated and the actual existing stress levels can be determined.

3.3.2. Type $B$ and $C$ Membrane Materials. As mentioned before, the developed membrane stress measurement equipment can be flexibly applied to all membrane materials, as it modifies only the respective mass $\rho_{k}$ of the targeted material. In this paper, not only Type $A$ but also Type $B$ and Type $C$ that 
TABLE 5: Result of the Type A membrane material under setup stress of $T_{x} / T_{y}$ or $T_{y} / T_{x}=C_{0}\left(C_{0}=\right.$ target ratio $)$.

\begin{tabular}{|c|c|c|c|c|c|c|c|c|c|c|}
\hline \multirow[t]{2}{*}{ Case } & \multicolumn{2}{|c|}{$\begin{array}{c}T_{x} / T_{y}=C_{0} \\
\left(C_{0}=\text { target ratio }\right) \\
\text { Setup stress }\left(\mathrm{kN} \mathrm{m}^{-1}\right)\end{array}$} & \multicolumn{2}{|c|}{$\begin{array}{l}\text { Estimation stress } \\
\qquad\left(\mathrm{kN} \mathrm{m}^{-1}\right)\end{array}$} & \multicolumn{2}{|c|}{$\begin{array}{l}\text { Estimation stress } \\
\qquad\left(\mathrm{kN} \mathrm{m}^{-1}\right)\end{array}$} & \multicolumn{2}{|c|}{$\begin{array}{l}\text { Error of stress } \\
\quad\left(\mathrm{kN} \mathrm{m}^{-1}\right)\end{array}$} & \multicolumn{2}{|c|}{ Error ratio (\%) } \\
\hline & $T_{x}$ & $T_{y}$ & $T_{x}$ & $T_{x}$ & $T_{x}$ & $T_{y}$ & $E_{x}$ & $E_{y}$ & $E_{x}$ & $E_{y}$ \\
\hline 21 & 1.69 & 1.94 & 1.40 & 1.52 & 1.70 & 1.84 & -0.01 & 0.10 & -0.59 & 5.15 \\
\hline 22 & 2.55 & 3.82 & 2.07 & 2.77 & 2.50 & 3.35 & 0.05 & 0.47 & 1.96 & 12.30 \\
\hline 23 & 3.44 & 5.57 & 2.52 & 4.03 & 3.04 & 4.87 & 0.40 & 0.70 & 11.63 & 12.57 \\
\hline 24 & 4.37 & 7.29 & 3.35 & 4.99 & 4.04 & 6.02 & 0.33 & 1.27 & 7.55 & 17.42 \\
\hline 25 & 5.39 & 9.20 & 4.09 & 5.87 & 4.93 & 7.06 & 0.46 & 2.14 & 8.53 & 23.26 \\
\hline 26 & 6.33 & 10.95 & 4.79 & 6.44 & 5.77 & 7.74 & 0.56 & 3.21 & 8.85 & 29.32 \\
\hline 27 & 7.25 & 12.94 & 5.28 & 7.05 & 6.35 & 8.46 & 0.90 & 4.48 & 12.41 & 34.62 \\
\hline 28 & 8.17 & 14.75 & 5.70 & 7.47 & 6.84 & 8.96 & 1.33 & 5.79 & 16.28 & 39.25 \\
\hline 29 & 9.15 & 16.88 & 6.06 & 7.59 & 7.27 & 9.10 & 1.88 & 7.78 & 20.55 & 46.09 \\
\hline 30 & 10.09 & 18.56 & 6.61 & 7.43 & 7.93 & 8.91 & 2.16 & 9.65 & 21.41 & 51.99 \\
\hline \multicolumn{11}{|c|}{$T_{y} / T_{x}=C_{0}$} \\
\hline 31 & 2.13 & 1.07 & 1.78 & 0.87 & 2.16 & 1.05 & -0.03 & 0.02 & -1.41 & 1.87 \\
\hline 32 & 4.02 & 1.98 & 3.13 & 1.52 & 3.79 & 1.85 & 0.23 & 0.13 & 5.72 & 6.57 \\
\hline 33 & 5.65 & 2.81 & 4.25 & 2.07 & 5.14 & 2.50 & 0.51 & 0.31 & 9.03 & 11.03 \\
\hline 34 & 7.62 & 3.79 & 5.38 & 2.81 & 6.49 & 3.39 & 1.13 & 0.40 & 14.83 & 10.55 \\
\hline 35 & 9.43 & 4.69 & 6.10 & 3.50 & 7.34 & 4.23 & 2.09 & 0.46 & 22.16 & 9.81 \\
\hline 36 & 11.49 & 5.76 & 6.83 & 4.24 & 8.21 & 5.10 & 3.28 & 0.66 & 28.55 & 11.46 \\
\hline 37 & 13.45 & 6.75 & 7.10 & 5.08 & 8.52 & 6.10 & 4.93 & 0.65 & 36.65 & 9.63 \\
\hline 38 & 15.48 & 7.75 & 7.54 & 5.61 & 9.04 & 6.74 & 6.44 & 1.01 & 41.60 & 13.03 \\
\hline 39 & 17.25 & 8.65 & 8.05 & 5.79 & 9.64 & 6.96 & 7.61 & 1.69 & 44.12 & 19.54 \\
\hline 40 & 19.37 & 9.70 & 8.17 & 6.17 & 9.78 & 7.41 & 9.59 & 2.29 & 49.51 & 23.61 \\
\hline Average & & & & & & & 2.19 & 2.16 & 17.97 & 27.22 \\
\hline Standard deviation & & & & & & & 2.72 & 2.66 & 14.67 & 13.69 \\
\hline
\end{tabular}

are currently being used in the construction sites are used in the experimental tests, in order to verify the expandability of the equipment.

The membrane materials of Type $\mathrm{B}$ and Type $\mathrm{C}$ that are used in the experimental tests have respective area densities of 0.81 and $0.87 \mathrm{~kg} \mathrm{~m}^{-2}$, which are measured by the same method as that of Type A. Introduced stress is established in three patterns as shown in Tables 6 and 7. The even stress of ratio $1: 1$ with stress levels of $0.5,1,1.5,2,3,4$, and $5 \mathrm{kN} \mathrm{m}^{-1}$ is introduced to both $X$ and $Y$ directions. Also, the different stresses of ratios $1: 2$ and $2: 1$ in $X$ and $Y$ directions are, respectively, introduced to Type $B$ and Type $C$. The introduced membrane stress is read from the force sensor when the tremor change of stress stops in the membrane after enough time has passed since the equipment has been placed on the membrane softly, similar to the experimental test for Type A.

Tables 6 and 7 show the results of the experimental tests using the membrane materials of Type $B$ and Type $C$ when the setup stresses are, respectively, $T_{x}=T_{y}=T_{0}\left(T_{0}=\right.$ target stress) and $T_{x} / T_{y}$ or $T_{y} / T_{x}=C_{0}\left(C_{0}=\right.$ target ratio $)$. Figures 13 and 14, respectively, represent the results of Tables 6 and 7 in a graph.

Compared to the experiment using Type A material, the tests using Type B and Type C materials show less accuracy in its results, when examining the same specific amount of stress level. Nonetheless, when the introduced stress is under $3 \mathrm{kN} \mathrm{m}^{-1}$, the experimental test using Type B and Type $\mathrm{C}$ shows approximately $70 \%$ accuracy. Such result can be explained by how the material hardens even with a comparatively low introduced stress, because Type B and Type $C$ are coated with a softer resin compared to Type A, thus making it difficult to divide the resonance boundary.

However, the developed equipment can be easily applied to various materials since it only modifies the respective mass, $\rho_{k}$, in (1) that has been suggested by the authors. Such approach is different from existing methods where each material needs a membrane stress level obtained in the laboratory in order to compare the estimation values. 


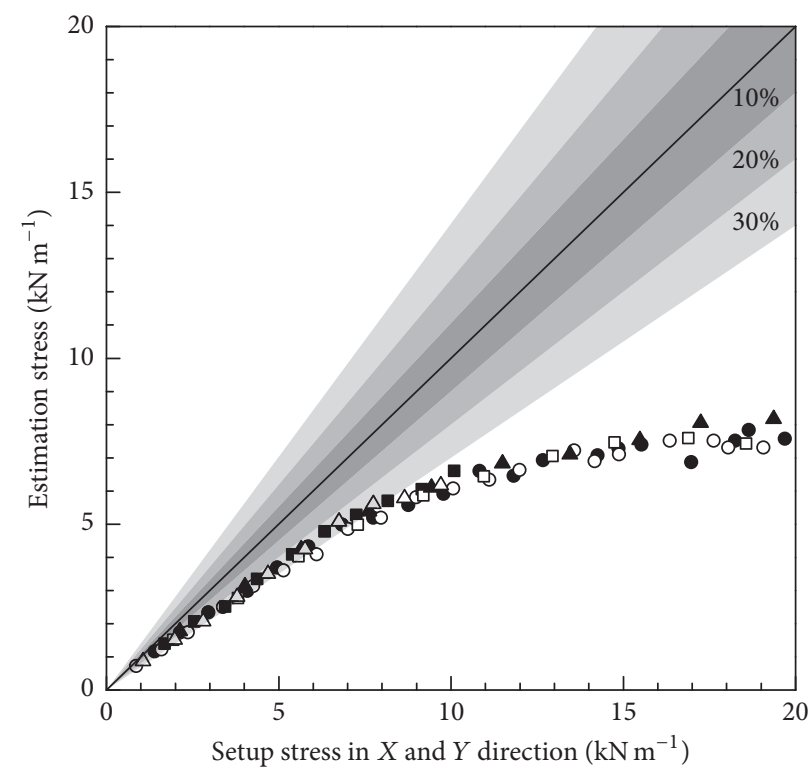

- $\left(T_{x}=T_{y}=T_{0}\right), X$ direction $\square\left(T_{x} / T_{y}=C_{0}\right), Y$ direction

○ $\left(T_{x}=T_{y}=T_{0}\right), Y$ direction $\Delta\left(T_{y} / T_{x}=C_{0}\right), X$ direction

- $\left(T_{x} / T_{y}=C_{0}\right), X$ direction $\Delta\left(T_{y} / T_{x}=C_{0}\right), Y$ direction

FIgURE 12: Result of membrane material Type A in the graph.

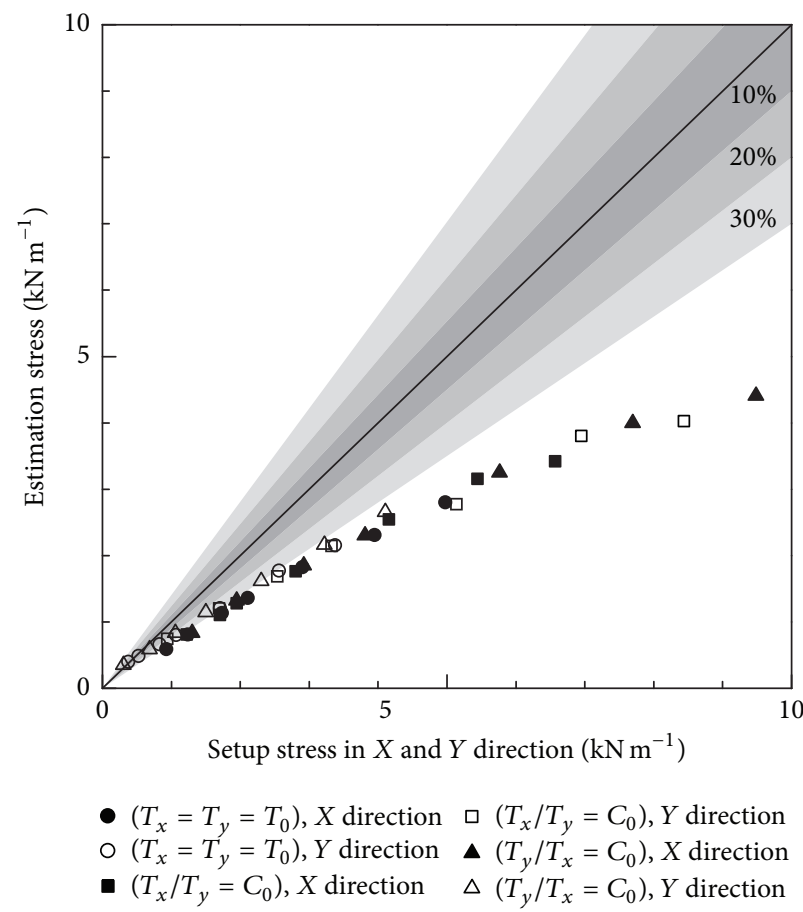

Figure 13: Result of membrane material of Type B in the graph.

\section{Evaluation of the Effect of Air}

When an object accelerates its motion within the fluids, the effect of fluids on an object is usually considered as added mass, which is equivalent to the additional mass corresponding to the kinetic energy of the flow moving with the object. The added mass is well-known in the field of fluid mechanics, and a number of books and research papers have been published in the field [9-19]. Usually the effect of air on the vibrating structures on the ground is not taken seriously. However, the effect of air on the vibrating membrane roof of the structure should not be neglected, because the mass 
TABLE 6: Result of the Type B membrane material under setup stress of $T_{x}=T_{y}=T_{0}$ and $T_{x} / T_{y}$ or $T_{y} / T_{x}=C_{0}\left(T_{0}=\right.$ target stress; $C_{0}=$ target ratio).

\begin{tabular}{|c|c|c|c|c|c|c|c|c|c|c|}
\hline \multirow[t]{2}{*}{ Case } & \multicolumn{2}{|c|}{$\begin{array}{c}T_{x}=T_{y}=T_{0} \\
\left(T_{0}=\text { target stress }\right) \\
\text { Setup stress }\left(\mathrm{kN} \mathrm{m}^{-1}\right)\end{array}$} & \multicolumn{2}{|c|}{$\begin{array}{l}\text { Estimation stress } \\
\qquad\left(\mathrm{kN} \mathrm{m}^{-1}\right)\end{array}$} & \multicolumn{6}{|c|}{ Considering added mass of membrane } \\
\hline & $T_{x}$ & $T_{y}$ & $T_{x}$ & $T_{x}$ & $T_{x}$ & $T_{y}$ & $E_{x}$ & $E_{y}$ & $E_{x}$ & $E_{y}$ \\
\hline 1 & 0.93 & 0.37 & 0.59 & 0.40 & 0.73 & 0.49 & 0.21 & -0.12 & 22.11 & -31.58 \\
\hline 2 & 1.24 & 0.52 & 0.81 & 0.49 & 1.00 & 0.60 & 0.24 & -0.08 & 19.05 & -15.09 \\
\hline 3 & 1.73 & 0.82 & 1.14 & 0.67 & 1.40 & 0.81 & 0.32 & 0.01 & 18.75 & 1.19 \\
\hline 4 & 2.11 & 1.07 & 1.32 & 0.80 & 1.68 & 0.99 & 0.43 & 0.08 & 20.47 & 7.34 \\
\hline 5 & 2.90 & 1.71 & 1.82 & 1.21 & 2.24 & 1.48 & 0.66 & 0.23 & 22.64 & 13.22 \\
\hline 6 & 3.95 & 2.56 & 2.31 & 1.77 & 2.82 & 2.18 & 1.13 & 0.38 & 28.54 & 14.93 \\
\hline 7 & 4.98 & 3.37 & 2.80 & 2.16 & 3.42 & 2.64 & 1.56 & 0.74 & 31.30 & 21.80 \\
\hline \multicolumn{11}{|c|}{$T_{x} / T_{y}=C_{0}$} \\
\hline 8 & 1.23 & 0.94 & 0.81 & 0.75 & 1.01 & 0.91 & 0.22 & 0.03 & 17.60 & 3.13 \\
\hline 9 & 1.71 & 1.70 & 1.11 & 1.21 & 1.36 & 1.48 & 0.34 & 0.22 & 20.12 & 12.72 \\
\hline 10 & 1.95 & 2.54 & 1.29 & 1.69 & 1.57 & 2.07 & 0.38 & 0.47 & 19.60 & 18.53 \\
\hline 11 & 2.80 & 3.32 & 1.76 & 2.15 & 2.17 & 2.63 & 0.64 & 0.70 & 22.73 & 20.94 \\
\hline 12 & 4.16 & 5.14 & 2.55 & 2.77 & 3.11 & 3.38 & 1.05 & 1.75 & 25.24 & 34.16 \\
\hline 13 & 5.44 & 6.95 & 3.16 & 3.80 & 3.84 & 4.64 & 1.60 & 2.31 & 29.37 & 33.27 \\
\hline 14 & 6.57 & 8.44 & 3.42 & 4.03 & 4.18 & 4.91 & 2.39 & 3.53 & 36.42 & 41.81 \\
\hline \multicolumn{11}{|c|}{$T_{y} / T_{x}=C_{0}$} \\
\hline 15 & 1.30 & 0.30 & 0.83 & 0.35 & 1.03 & 0.43 & 0.27 & -0.13 & 21.05 & -41.94 \\
\hline 16 & 1.95 & 0.69 & 1.32 & 0.59 & 1.68 & 0.72 & 0.32 & -0.03 & 16.58 & -4.29 \\
\hline 17 & 2.92 & 1.06 & 1.85 & 0.83 & 2.26 & 1.02 & 0.66 & 0.04 & 22.48 & 3.70 \\
\hline 18 & 3.81 & 1.50 & 2.30 & 1.15 & 2.81 & 1.40 & 1.00 & 0.10 & 26.22 & 6.54 \\
\hline 19 & 5.76 & 2.30 & 3.25 & 1.62 & 3.98 & 1.98 & 1.78 & 0.32 & 30.95 & 14.04 \\
\hline 20 & 7.69 & 3.22 & 4.00 & 2.17 & 4.87 & 2.65 & 2.82 & 0.58 & 36.67 & 17.93 \\
\hline 21 & 9.49 & 4.11 & 4.41 & 2.66 & 5.37 & 3.24 & 4.12 & 0.86 & 43.39 & 21.02 \\
\hline Average & & & & & & & 1.05 & 0.57 & 25.30 & 9.21 \\
\hline Standard deviation & & & & & & & 1.00 & 0.90 & 6.99 & 19.70 \\
\hline
\end{tabular}

of membrane per area is as light as the surrounding air contacting the vibrating membrane.

In a number of studies, Minami derived a ratio by adding the mass of air to the mass of the vibrating membrane itself at a finite amplitude, $\alpha$, which is equal to 0.68 divided by the mass ratio $m /\left(\rho_{0} l\right), m$ is the mass per unit area of the membrane itself, $\rho_{0}$ is the density of the air flow, and $l$ is the length of the membrane. He did this by applying the thin aerofoil theory where the membrane is modeled as a vortex sheet. Also, the added mass is equal to the mass of air in a rectangle with a height of $0.68 l$ over the membrane, regardless of the period and amplitude of the oscillation [20].

In this paper, the proposed method for estimating membrane stress is used to measure the natural frequency of a vibrating membrane. At every stress level, the influence of the air surrounding the membrane that vibrates with various amplitudes is theoretically evaluated in an acoustic impedance approach. Here, the vibration of the rectangular plate can be assumed to be replaced by a vibrating flat circular plate of the same surface area.

The effect that the intermediate medium surrounding the plate has on the vibration can be evaluated as the sum of the point sources' effect that is scattered in the intermediate medium when the plate is vibrating. The acoustic radiation in the intermediate medium that is generated by the vibration of the plate produces acoustic pressure on the vibrating plate to repress its vibration. Therefore, extra forces are needed to keep the plate vibrating at the same velocity as in the vacuum condition [21-23]. It is assumed that the rigid circular plate vibrates in a rigid infinite baffle and that each point on the plane surface may be considered as a source of hemispherical waves. When the circular plate is moving at a complex velocity, a quantity $Z_{r}$, called the radiation impedance of the circular plate and defined as the ratio of the force exerted by 
TABLE 7: Result of the Type C membrane material under setup stress of $T_{x}=T_{y}=T_{0}$ and $T_{x} / T_{y}$ or $T_{y} / T_{x}=C_{0}\left(T_{0}=\operatorname{target}\right.$ stress; $C_{0}=\operatorname{target}$ ratio).

\begin{tabular}{|c|c|c|c|c|c|c|c|c|c|c|}
\hline \multirow{3}{*}{ Case } & \multirow{2}{*}{\multicolumn{2}{|c|}{$\begin{array}{c}T_{x}=T_{y}=T_{0} \\
\left(T_{0}=\text { target stress }\right) \\
\text { Setup stress }\left(\mathrm{kN} \mathrm{m}^{-1}\right)\end{array}$}} & \multirow{2}{*}{\multicolumn{2}{|c|}{$\begin{array}{l}\text { Estimation stress } \\
\qquad\left(\mathrm{kN} \mathrm{m}^{-1}\right)\end{array}$}} & \multicolumn{6}{|c|}{ Considering added mass of membrane } \\
\hline & & & & & \multicolumn{2}{|c|}{$\begin{array}{l}\text { Estimation stress } \\
\qquad\left(\mathrm{kN} \mathrm{m}^{-1}\right)\end{array}$} & \multicolumn{2}{|c|}{$\begin{array}{l}\text { Error of stress } \\
\qquad\left(\mathrm{kN} \mathrm{m}^{-1}\right)\end{array}$} & \multicolumn{2}{|c|}{ Error ratio (\%) } \\
\hline & $T_{x}$ & $T_{y}$ & $T_{x}$ & $T_{x}$ & $T_{x}$ & $T_{y}$ & $E_{x}$ & $E_{y}$ & $E_{x}$ & $E_{y}$ \\
\hline 1 & 0.67 & 0.57 & 0.56 & 0.42 & 0.69 & 0.51 & -0.02 & 0.06 & -2.94 & 10.34 \\
\hline 2 & 1.11 & 0.78 & 0.83 & 0.56 & 1.02 & 0.69 & 0.09 & 0.10 & 7.97 & 12.50 \\
\hline 3 & 1.53 & 1.12 & 1.09 & 0.78 & 1.34 & 0.97 & 0.19 & 0.15 & 12.18 & 13.16 \\
\hline 4 & 1.95 & 1.48 & 1.37 & 1.01 & 1.69 & 1.25 & 0.26 & 0.23 & 13.57 & 15.23 \\
\hline 5 & 2.83 & 2.33 & 1.84 & 1.52 & 2.26 & 1.86 & 0.57 & 0.47 & 20.07 & 20.17 \\
\hline 6 & 3.70 & 3.19 & 2.33 & 2.00 & 2.85 & 2.45 & 0.85 & 0.74 & 23.02 & 23.08 \\
\hline 7 & 4.62 & 4.13 & 2.70 & 2.54 & 3.31 & 3.11 & 1.30 & 1.02 & 28.24 & 24.70 \\
\hline \multicolumn{11}{|c|}{$T_{x} / T_{y}=C_{0}$} \\
\hline 8 & 1.06 & 1.06 & 0.76 & 0.74 & 0.93 & 0.92 & 0.13 & 0.14 & 12.04 & 12.96 \\
\hline 9 & 1.45 & 1.87 & 1.01 & 1.25 & 1.23 & 1.54 & 0.22 & 0.32 & 14.87 & 17.37 \\
\hline 10 & 1.85 & 2.75 & 1.25 & 1.78 & 1.55 & 2.19 & 0.30 & 0.57 & 16.40 & 20.64 \\
\hline 11 & 2.34 & 3.67 & 1.57 & 2.25 & 1.92 & 2.76 & 0.42 & 0.90 & 17.99 & 24.60 \\
\hline 12 & 3.19 & 5.27 & 2.20 & 2.93 & 2.70 & 3.59 & 0.49 & 1.69 & 15.39 & 31.97 \\
\hline 13 & 4.08 & 6.91 & 2.80 & 3.65 & 3.42 & 4.45 & 0.66 & 2.46 & 16.11 & 35.60 \\
\hline 14 & 4.96 & 8.49 & 2.96 & 4.25 & 3.62 & 5.18 & 1.34 & 3.30 & 27.08 & 38.92 \\
\hline \multicolumn{11}{|c|}{$T_{y} / T_{x}=C_{0}$} \\
\hline 15 & 1.16 & 0.49 & 0.84 & 0.34 & 1.03 & 0.42 & 0.13 & 0.07 & 11.02 & 14.00 \\
\hline 16 & 1.95 & 0.91 & 1.38 & 0.62 & 1.70 & 0.75 & 0.25 & 0.16 & 13.07 & 17.20 \\
\hline 17 & 2.88 & 1.37 & 1.89 & 0.96 & 2.32 & 1.18 & 0.56 & 0.20 & 19.39 & 14.29 \\
\hline 18 & 3.80 & 1.85 & 2.39 & 1.27 & 2.93 & 1.56 & 0.87 & 0.29 & 22.94 & 15.87 \\
\hline 19 & 5.60 & 2.79 & 3.17 & 1.95 & 3.87 & 2.39 & 1.72 & 0.40 & 30.82 & 14.39 \\
\hline 20 & 7.26 & 3.67 & 3.99 & 2.26 & 4.88 & 2.76 & 2.38 & 0.90 & 32.79 & 24.60 \\
\hline 21 & 8.78 & 4.60 & 4.38 & 2.82 & 5.34 & 3.45 & 3.44 & 1.15 & 39.17 & 24.95 \\
\hline Average & & & & & & & 0.73 & 0.77 & 20.31 & 18.63 \\
\hline Standard deviation & & & & & & & 0.84 & 0.84 & 7.69 & 9.23 \\
\hline
\end{tabular}

the circular plate on the medium to the velocity of the circular plate, becomes involved. From it, we obtain the following equation:

$$
Z_{r}=\rho_{0} c \pi a^{2}\left(R_{1}(2 k a)+j X_{1}(2 k a)\right),
$$

where $\rho_{0}$ is the density of the medium, $c$ is the speed of sound, $a$ is the radius of the vibrating plate, $j=\sqrt{-1}$, and $R_{1}(2 k a)$ and $X_{1}(2 k a)$ are the power series shown as follows:

$$
\begin{aligned}
& R_{1}(2 k a)=1-\frac{2 J_{1}(2 k a)}{2 k a} \\
& =\left(\frac{(2 k a)^{2}}{2 \cdot 4}-\frac{(2 k a)^{4}}{2 \cdot 4^{2} \cdot 6}+\frac{(2 k a)^{6}}{2 \cdot 4^{2} \cdot 6^{2} \cdot 8}-\cdots\right), \\
& X_{1}(2 k a)=\frac{2 K_{1}(2 k a)}{(2 k a)^{2}} \\
& =\frac{4}{\pi}\left(\frac{(2 k a)}{3}-\frac{(2 k a)^{3}}{3^{2} \cdot 5}+\frac{(2 k a)^{5}}{3^{2} \cdot 5^{2} \cdot 7}-\cdots\right),
\end{aligned}
$$

where $J_{1}$ represents a Bessel function of the first kind and $K_{1}$ also represents a modified Bessel function of the first kind. The radiation resistance has a mass dimension related to acceleration. Its effect is equivalent to simply adding the actual mass of the circular plate to $\Delta m$, as follows:

$$
\Delta m=\frac{X_{r}}{\omega}=\frac{X_{r}}{k c}=\rho_{0} \pi a^{2} \frac{X_{1}(2 k a)}{k} .
$$

Figure 15 shows that the added mass of the membrane with the effect of air on the vibrating circular plate can be approximately evaluated by the same rectangular plate area of $200 \mathrm{~mm} \times 300 \mathrm{~mm}$, which is the size of the vibrating membrane boundary in the measurement equipment. As shown in Figure 15, the added mass of membrane is the function of frequency, and the magnitude of the added mass decreases as the frequency increases. However, the added mass should not be neglected when it is within the low frequency range, and this added mass of membrane has a fairly constant value. In this case, the added mass 


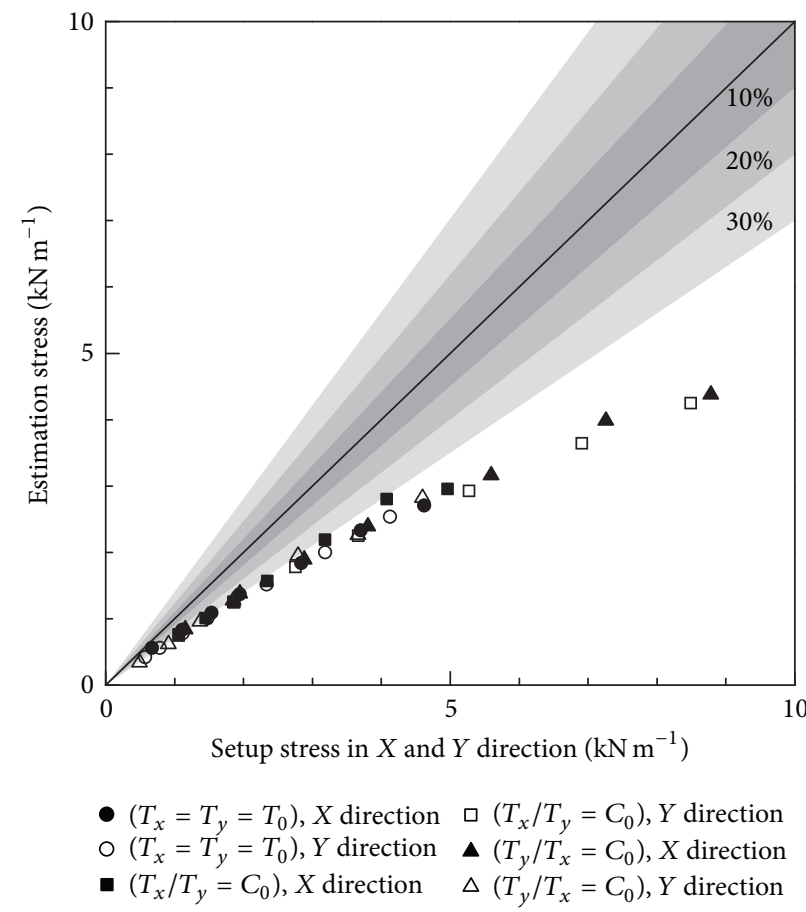

Figure 14: Result of membrane material of Type $C$ in the graph.

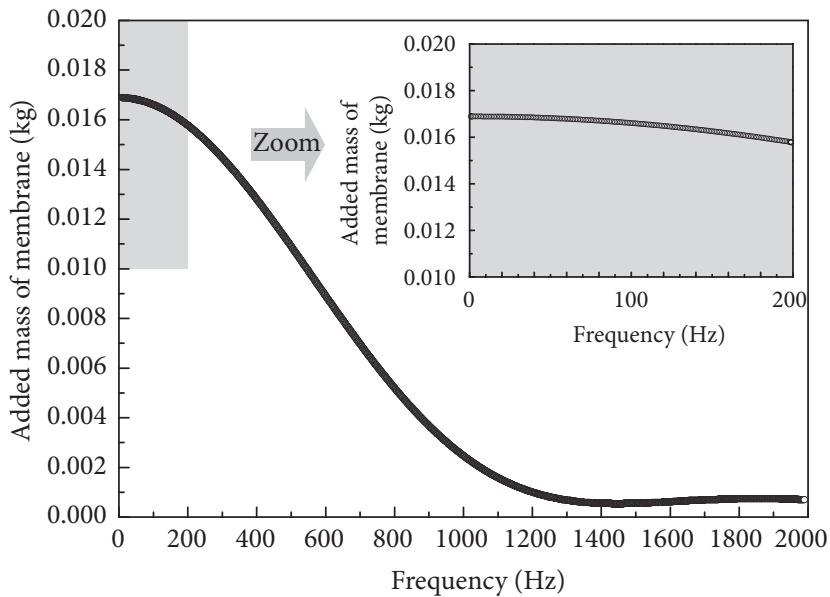

FIGURE 15: Added mass of membrane in effect to air.

of membrane values is around $0.017 \mathrm{~kg}$, as compared with $0.015 \mathrm{~kg}$ of the Minami added mass [20]. The differences are due to boundary conditions, the form of vibration, and the method of analysis.

All the vibrating mass per unit area, $\rho_{\text {all }}$, would be expressed as $\rho_{k}+\Delta m$ in the proposed equation (1) for the membrane stress. Tables $4,5,6$, and 7 show the results considering the added mass of each kind of membrane materials with the effect of air as mentioned above, by substituting $\rho_{\text {all }}$ for the $\rho_{k}$ in (1). Also, the " $x$ " symbols in Figures 16, 17, and 18 show the results in a graph. It is confirmed that, by considering the added mass of each membrane for the effect of air, the estimation accuracy of all the membrane used in the experiment increases about $20 \%$ in comparison to the original estimation stress that covers all of the introduced stress levels.

\section{Error Correction}

Even when the effect of air with the added mass of membrane has been considered, the measurement errors increase when the introduced stresses increase, as those described by the quadratic function shown in Figures 19, 20, and 21. While considering the added mass of the membrane, there are 


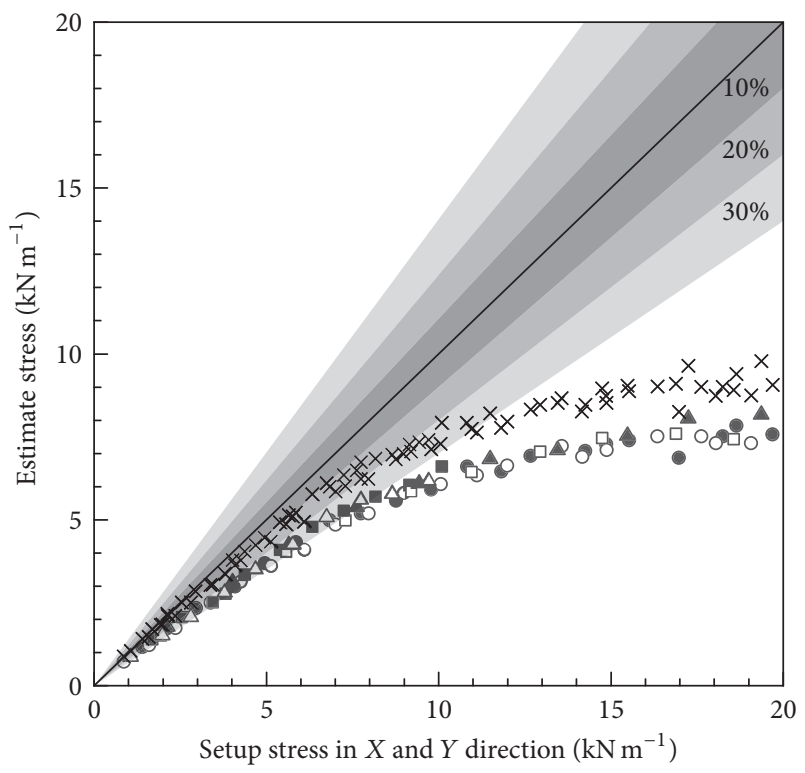

- $\left(T_{x}=T_{y}=T_{0}\right), X$ direction $\square\left(T_{x} / T_{y}=C_{0}\right), Y$ direction

○ $\left(T_{x}=T_{y}=T_{0}\right), Y$ direction $\Delta\left(T_{y} / T_{x}=C_{0}\right), X$ direction

- $\left(T_{x} / T_{y}=C_{0}\right), X$ direction $\Delta\left(T_{y} / T_{x}=C_{0}\right), Y$ direction

$\times$ With added mass

FIGURE 16: Result considering added mass of membrane material of Type A.

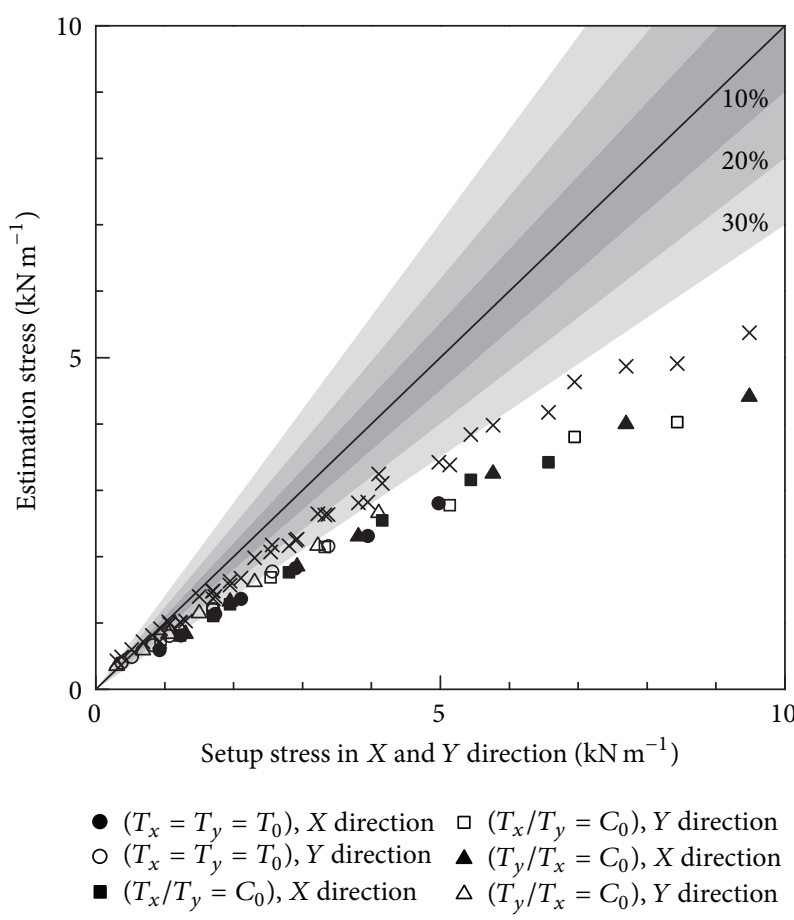

FIGURE 17: Result considering added mass of membrane material of Type B.

other errors that are difficult to inspect with our estimation method induced by the equation of membrane oscillation, such as the damping of the membrane, added damping of the membrane from air, and other unexplained errors.

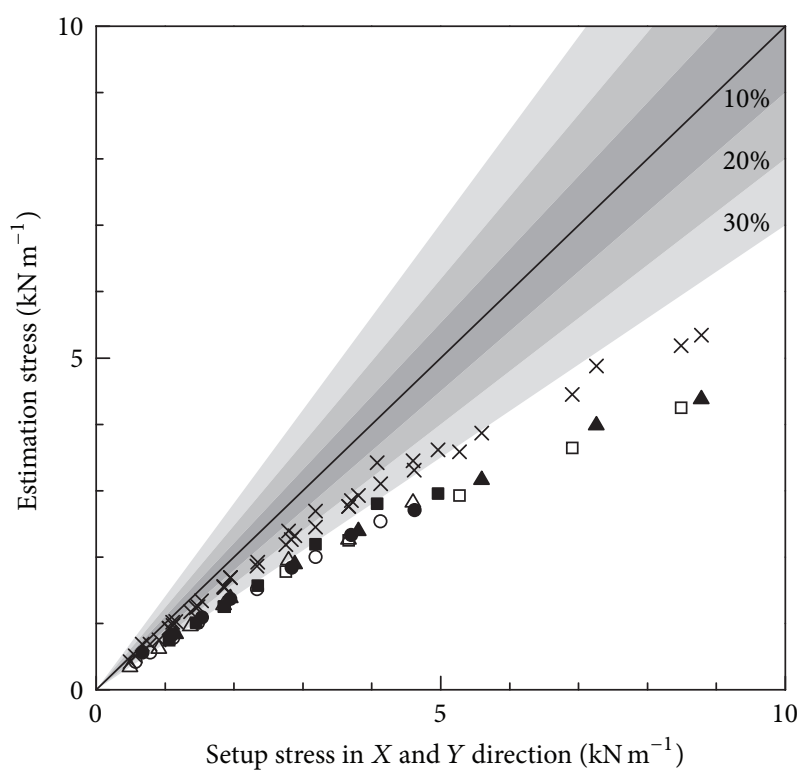

- $\left(T_{x}=T_{y}=T_{0}\right), X$ direction $\square\left(T_{x} / T_{y}=C_{0}\right), Y$ direction

○ $\left(T_{x}=T_{y}=T_{0}\right), Y$ direction $\Delta\left(T_{y} / T_{x}=C_{0}\right), X$ direction

- $\left(T_{x} / T_{y}=C_{0}\right), X$ direction $\Delta\left(T_{y} / T_{x}=C_{0}\right), Y$ direction

FIGURE 18: Result considering added mass of membrane material of Type C.

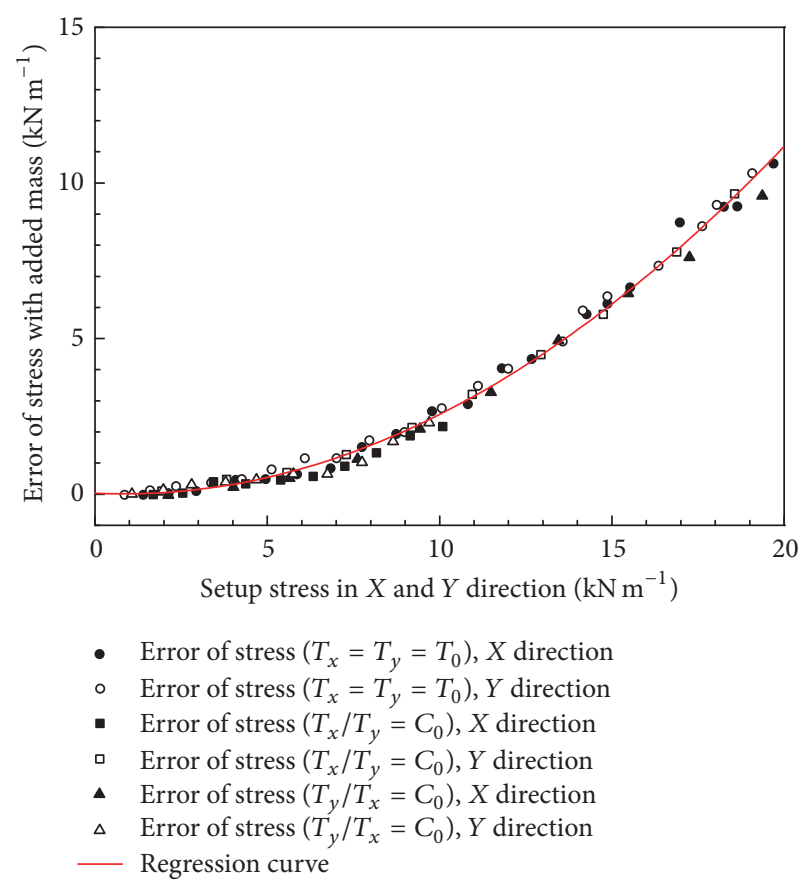

FIGURE 19: Error regression analysis of membrane material of Type A.

For that reason, regression analysis is carried out using a polynomial expression in order to correct the errors from the experimental tests. Symbols in Figures 19, 20, and 21 indicate the errors in all stress levels of the $X$ and $Y$ directions that are introduced in the experimental tests, and the curved lines 


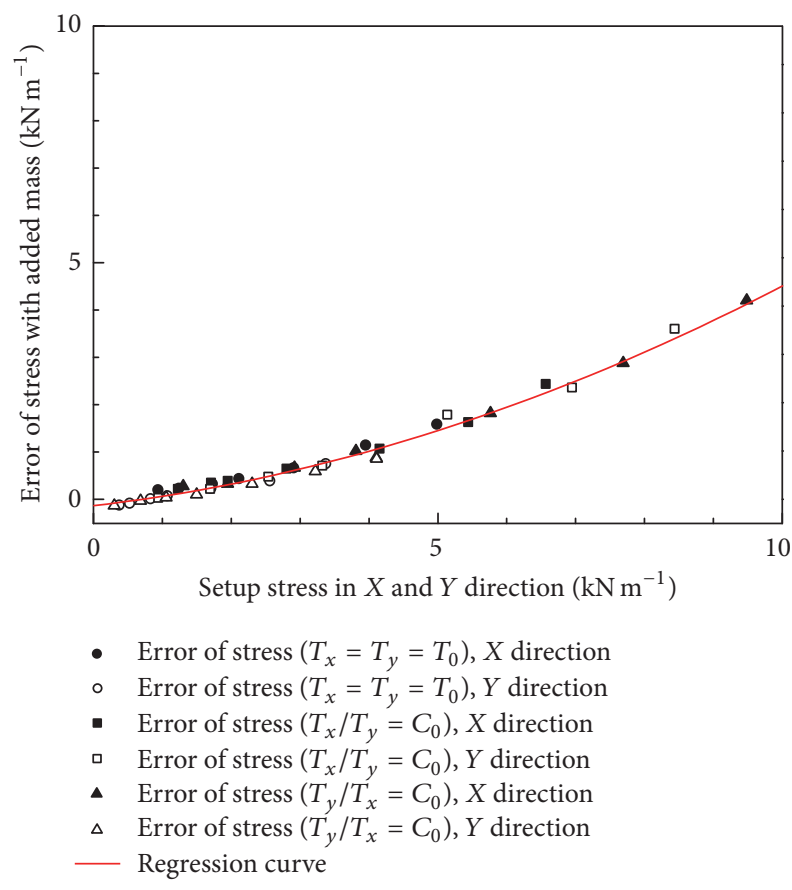

FIGURE 20: Error regression analysis of membrane material of Type B.

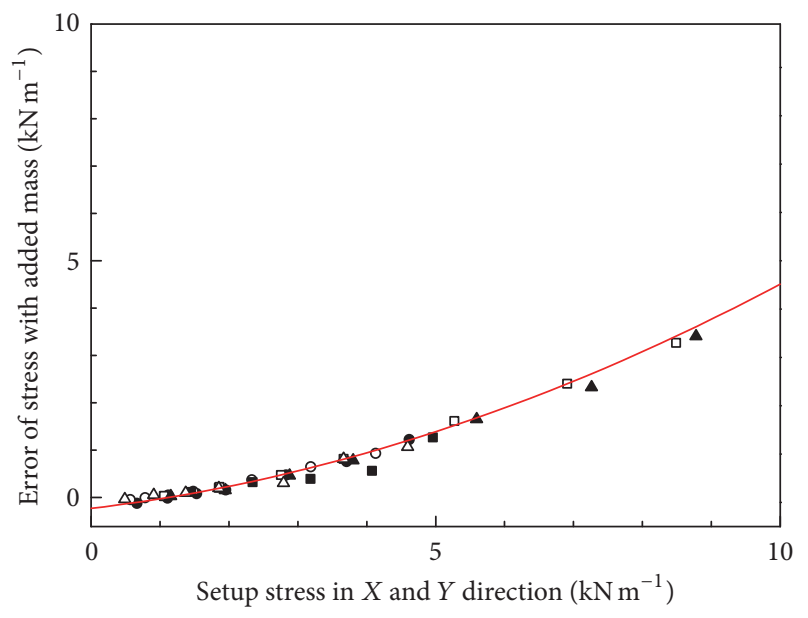

- Error of stress $\left(T_{x}=T_{y}=T_{0}\right), X$ direction

- Error of stress $\left(T_{x}=T_{y}=T_{0}\right), Y$ direction

- Error of stress $\left(T_{x} / T_{y}=C_{0}\right), X$ direction

- Error of stress $\left(T_{x} / T_{y}=C_{0}\right), Y$ direction

- Error of stress $\left(T_{y} / T_{x}=C_{0}\right), X$ direction

$\Delta \quad$ Error of stress $\left(T_{y} / T_{x}=C_{0}\right), Y$ direction

- Regression curve

FIGURE 21: Error regression analysis of membrane material of Type C.

indicate the result of the regression curve obtained using the quadratic expression.

Table 8 shows the coefficients of the regression curves. The determination coefficient indicates the square of the correlation coefficient obtained in the regression analysis and also shows how much can be explained by the regression

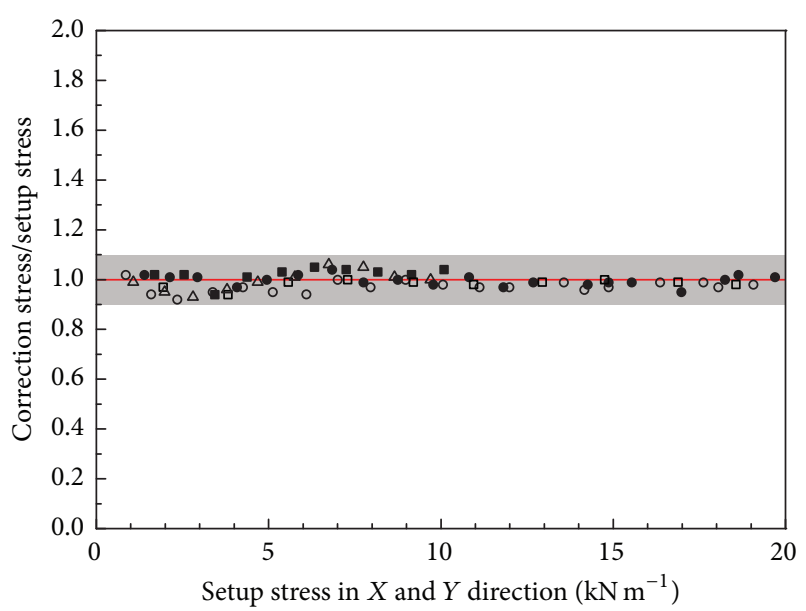
- $T_{x}=T_{y}=T_{0}, X$ direction
- $T_{x}=T_{y}=T_{0}, Y$ direction
- $T_{x} / T_{y}=C_{0}, Y$ direction
- $T_{x} / T_{y}=C_{0}, X$ direction
$\Delta T_{y} / T_{x}=C_{0}, X$ direction
$\Delta T_{y} / T_{x}=C_{0}, Y$ direction

FIGURE 22: Error correction of membrane material of Type A.

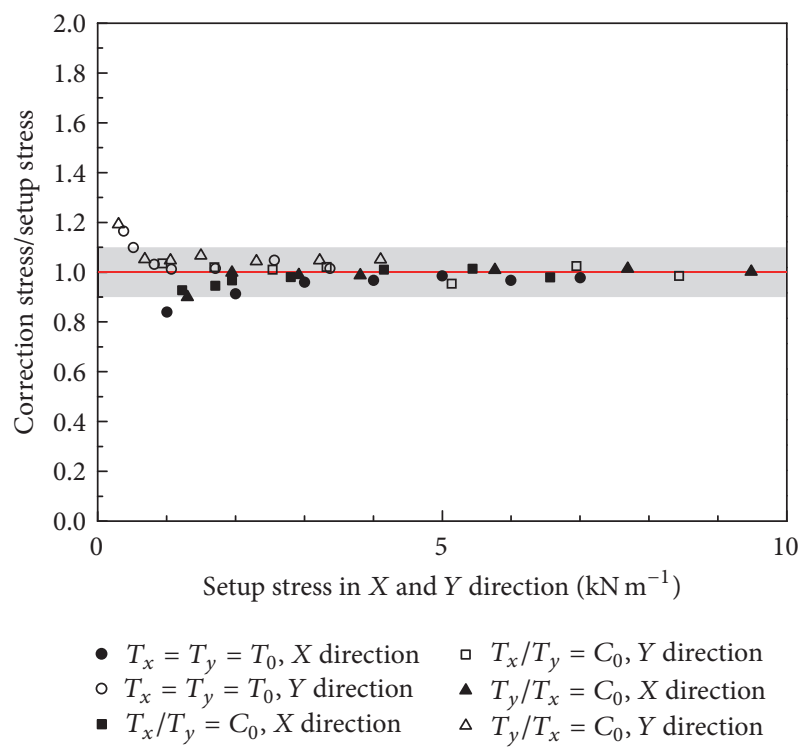

FIGURE 23: Error correction of membrane material of Type B.

curve from the total amount of fluctuation. The standard deviations show the relationship between the errors estimated by both the results of the data and the real error. Therefore, a determination coefficient of 0.9940 explains $99.40 \%$ of all fluctuations of the object function to the regression curve. Figures 22, 23, and 24 show the results obtained by the correction errors of the each membrane. The estimation's accuracy of error under $\pm 10 \%$ is obtained with the error correction in the range of the membrane stress introduced.

\section{Conclusions}

In this paper, a basic concept for a new method of estimating membrane stress with high accuracy is presented. Such new 
TABLE 8: Coefficients of the regression curve for each membrane material.

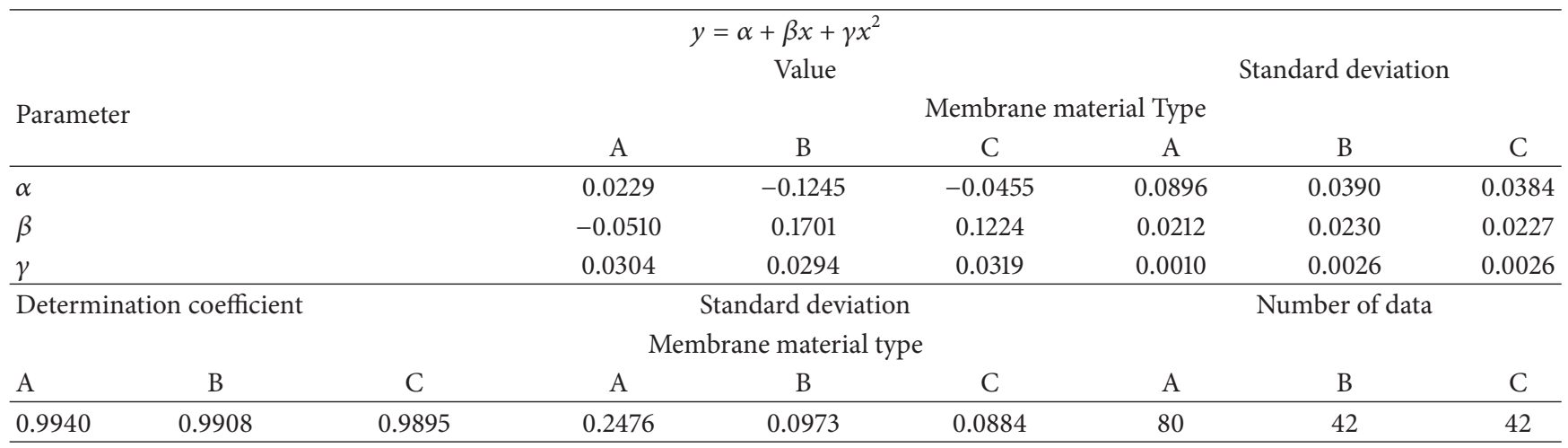

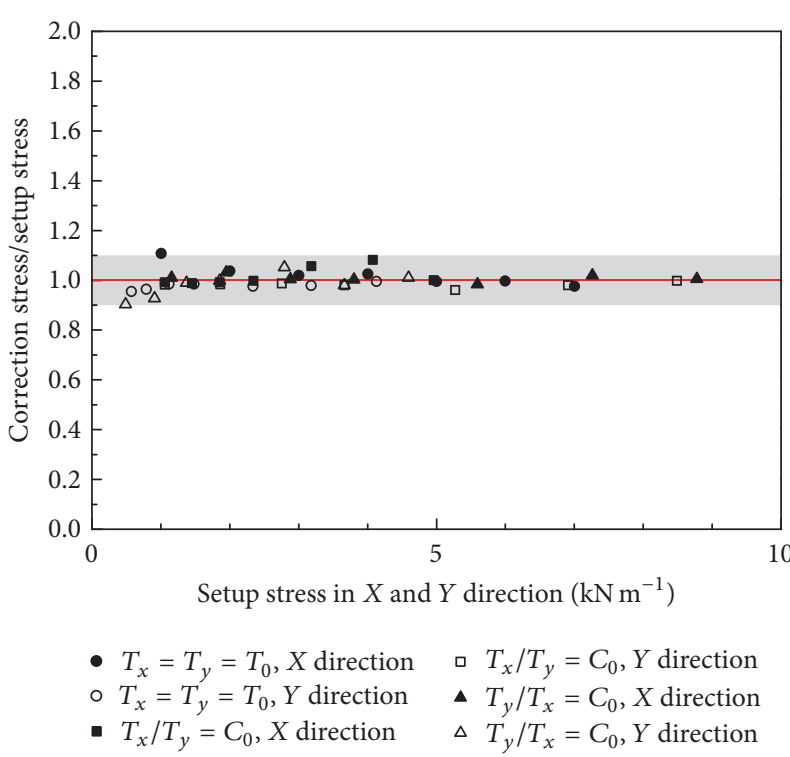

FIgURE 24: Error correction of membrane material of Type C.

method is based on the resonance phenomenon of the membranes induced by sound waves.

The measurement equipment is improved to ensure stable accuracy. Such equipment is experimented in order to verify the expandability and further accuracy using three membrane materials made of one kind of PTFE-coated glass fiber, PVC-coated glass fiber, and PVC-coated synthetic fiber. Such experiments record two different frequencies under the application of white noise sound waves from a speaker to produce vibrations on the membrane. Using improved measurement equipment in the membrane tensile force tests, it is confirmed that the measurement error can be maintained at less than $30 \%$ in Type A membrane material with the range of an introduced stress of under $5 \mathrm{kN} \mathrm{m}^{-1}$ and Type $\mathrm{B}$ and $\mathrm{C}$ materials of under $3 \mathrm{kN} \mathrm{m}^{-1}$.

The added mass of membrane with the effect of air is theoretically considered by assuming that the radiation impedance of the rectangular plate could be approximately evaluated by the results obtained from a circular plate of the same area. It is also confirmed that though the function of the added mass is frequency-dependent, the values are constant within the low frequency range. In this case, the added mass of membrane values is around $0.017 \mathrm{~kg}$, which is nearly equal to the added mass value obtained by Minami. In the membrane vibration, the effect of air cannot be neglected, and approximately $20 \%$ improvement in estimation accuracy on all membrane materials is obtained by considering the added mass of membrane with the effect of air.

The error according to the introduced stress level behaves like an increasing quadratic function, after the correction of measurement errors that includes unexplained errors. Using regression analysis, the accuracy within a tolerance of under $\pm 10 \%$ is obtained.

The tested measurements proved the possibility of continuing experimentation with the use of white noise sound waves as a tool to scientifically and quantitatively determine the membrane stress.

In the future, not only the downsizing of equipment but also the application of such equipment to preexisting membrane structures should be considered in order to obtain practical use.

\section{Nomenclature}

$T_{x}, T_{y}$ : Existing tensions per length in $x$ and $y$ $T_{x} y: \quad$ Shear force in woven membrane material $m_{1,2}, n_{1,2}: \quad$ Number of waves in the vibration mode $a_{1}, b_{1}, a_{2}, b_{2}$ : Side lengths of the rectangular boundary when the external excitation with the respective frequencies $f_{1}$ and $f_{2}$ is observed

$f_{1}, f_{2}: \quad$ Resonance frequencies

$\rho_{k}: \quad \quad$ Membrane mass per unit area

A: $\quad$ Membrane material of Type A

B: $\quad$ Membrane material of Type B

C: $\quad$ Membrane material of Type C

$T_{0}$ : $\quad$ Target stress

$C_{0}=T_{y} / T_{x}:$ Target ratio 

$\alpha$ : $\quad$ Minami derived from a ratio by adding the mass of air to the mass of the vibrating membrane itself at finite amplitude
$\rho_{0}: \quad$ Density of the air flow
$l: \quad$ Length of the membrane
$Z_{r}$ : $\quad$ Radiation impedance of the circular plate
$c$ : $\quad$ Speed of sound
$a$ : Radius of the vibrating plate
$R_{1}, X_{1}$ : Power series
$J_{1}$ : $\quad$ Bessel function of the first kind
$K_{1}$ : $\quad$ Modified Bessel function of the first kind
$\Delta m: \quad$ Added mass of the circular plate
$\rho_{\text {all }}$ : All the vibrating mass per unit area.

\section{Conflicts of Interest}

The authors declare that they have no conflicts of interest.

\section{Acknowledgments}

This research was supported not only by Basic Science Research Program through the National Research Foundation of Korea (NRF) funded by the Ministry of Science, ICT \& Future Planning (NRF-2014R1A2A1A01004473) and a Grant (17AUDP-B100343-03) from Architecture \& Urban Development Research Program funded by Ministry of Land, Infrastructure and Transport of Korean government, but also by the Graduate School Research Program of Korea Tech.

\section{References}

[1] N. Sasaki and H. Tsubota, "Development of measurement system of membrane stress," Summaries of Technical Papers of Annual Meeting of AIJ, pp. 1389-1390, 1993.

[2] N. Sasaki and H. Tsubota, "Simple film-tension measuring instrument," Bulletin of Patent and Utility Model in Japan Patent Office, 1993.

[3] S. Yuzuba, N. Ishizu, and T. Taga, "Membrane stress measurement device for membrane structures," Bulletin of Patent and Utility Model in Japan Patent Office, 1994.

[4] H.-M. Jung, J.-W. Woo, B.-W. Cho, and S.-Y. Lee, "Development of measuring system of membrane stress for membrane structure," Journal of the Korean Association for Spatial Structures, vol. 8, no. 6, pp. 67-74, 2008.

[5] K. Tanizawa, "Measurement device of membrane stress," Bulletin of Patent and Utility Model in Japan Patent Office, 1992.

[6] N. Sasaki, "Measurement device of membrane stress," Bulletin of Patent and Utility Model in Japan Patent Office, 1994.

[7] S.-W. Jin and H. Ohmori, "Development of membrane stress measurement equipment for membrane structures: proposal of measuring method and its experimental verification," Measurement Science and Technology, vol. 22, no. 11, article 115704, 2011.

[8] http://www.keyence.com/products/measure/laser/lkg/lkg.php.

[9] H. Lamb, Hydrodynamics, Cambridge Mathematical Library, Cambridge University Press, Cambridge, UK, 6th edition, 1932.

[10] R. W. Arnold and G. B. Warburton, "The flexural vibration of thin cylinders," Proceedings of the Royal Society of London A, vol. 197, pp. 238-256, 1948.
[11] B. Nath, "On the first invariant of the virtual mass tensor," Journal of Physics D: Applied Physics, vol. 2, no. 6, article 421, pp. 924-928, 1969.

[12] FRITZ RJ, "Effect of liquids on the dynamic motions of immersed solids," Journal of Engineering for Industry ASME Series B, vol. 94, no. 1, pp. 167-173, 1972.

[13] P. R. Payne, "The virtual mass of a rectangular flat plate of finite aspect ratio," Ocean Engineering, vol. 8, no. 5, pp. 541-545, 1981.

[14] C. T. F. Ross and T. Johns, "Vibration of circular cylinders containing water," Thin-Walled Structures, vol. 12, no. 3, pp. 177196, 1991.

[15] M. R. Maheri and R. T. Severn, "Experimental added-mass in modal vibration of cylindrical structures," Engineering Structures, vol. 14, no. 3, pp. 163-175, 1992.

[16] B. W. Northway, N. H. Hancock, and T. Tran-Cong, "Liquid level sensors using thin walled cylinders vibrating in circumferential modes," Measurement Science and Technology, vol. 6, no. 1, article 014, pp. 85-93, 1995.

[17] H. Koivurova and A. Pramila, "Nonlinear vibration of axially moving membrane by finite element method," Computational Mechanics, vol. 20, no. 6, pp. 573-581, 1997.

[18] C. G. Rodriguez, E. Egusquiza, X. Escaler, Q. W. Liang, and F. Avellan, "Experimental investigation of added mass effects on a Francis turbine runner in still water," Journal of Fluids and Structures, vol. 22, no. 5, pp. 699-712, 2006.

[19] Y. Kerboua, A. A. Lakis, M. Thomas, and L. Marcouiller, "Vibration analysis of rectangular plates coupled with fluid," Applied Mathematical Modelling, vol. 32, no. 12, pp. 2570-2586, 2008.

[20] H. Minami, "Added mass of a membrane vibrating at finite amplitude," Journal of Fluids and Structures, vol. 12, no. 7, pp. 919-932, 1998.

[21] L. E. Kinsler and A. R. Fery, Fundamentals of Acoustics, John Wiley \& Sons, New York, NY, USA, 1962.

[22] R. W. B. Stephens and A. E. Bate, Acoustics and Vibrational Physics, Edward Arnold, London, UK, 1966.

[23] P. M. Morse and K. U. Ingard, Theoretical Acoustics, McGrawHill, New York, NY, USA, 1968. 


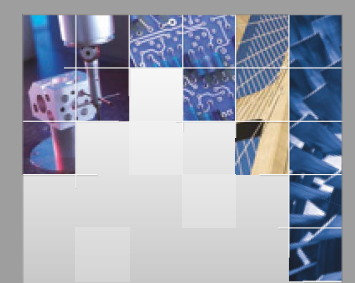

\section{Enfincering}
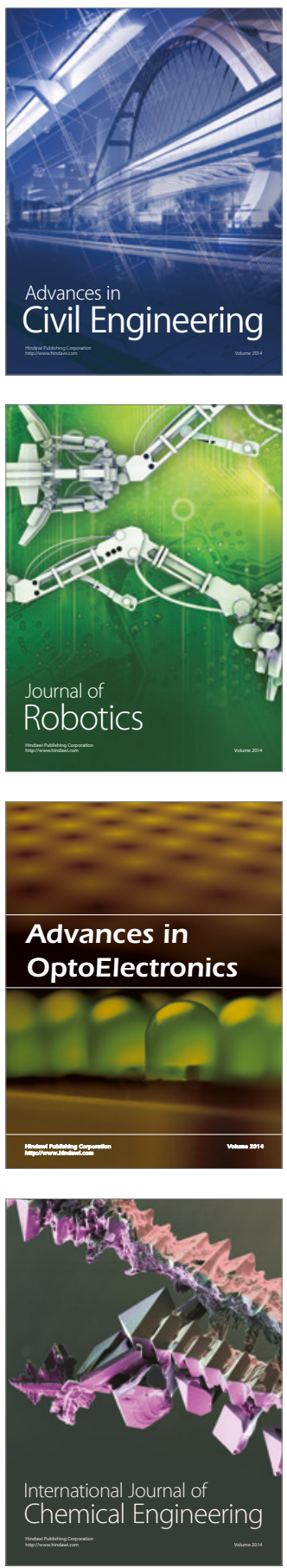

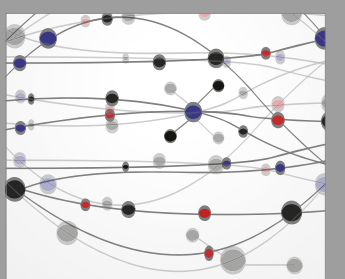

The Scientific World Journal

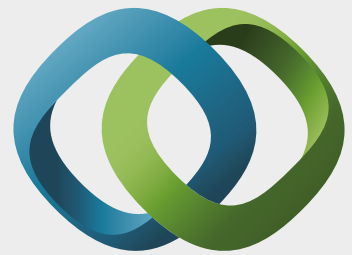

\section{Hindawi}

Submit your manuscripts at

https://www.hindawi.com
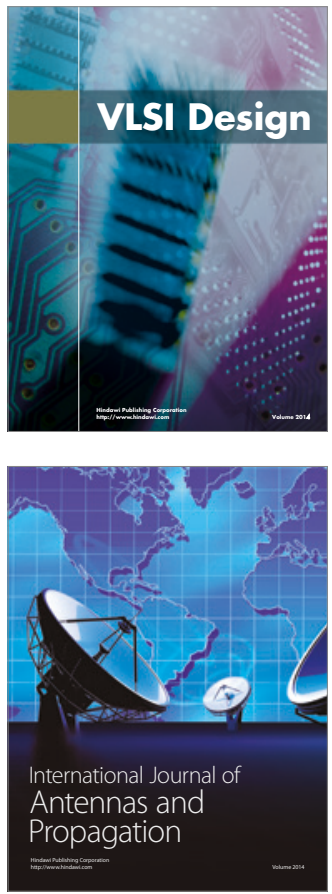

\section{Rotating}

Machinery
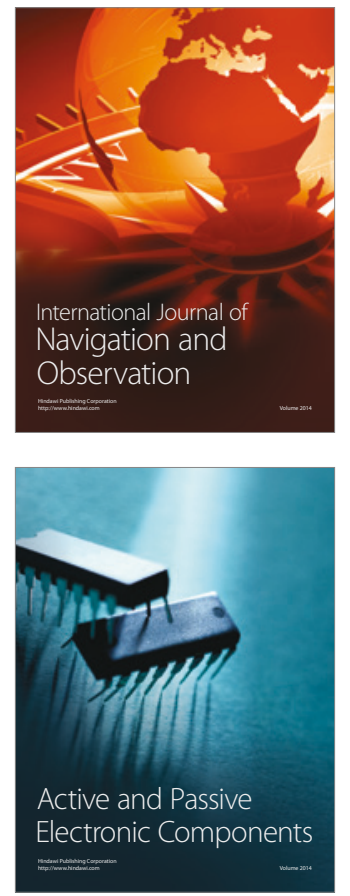
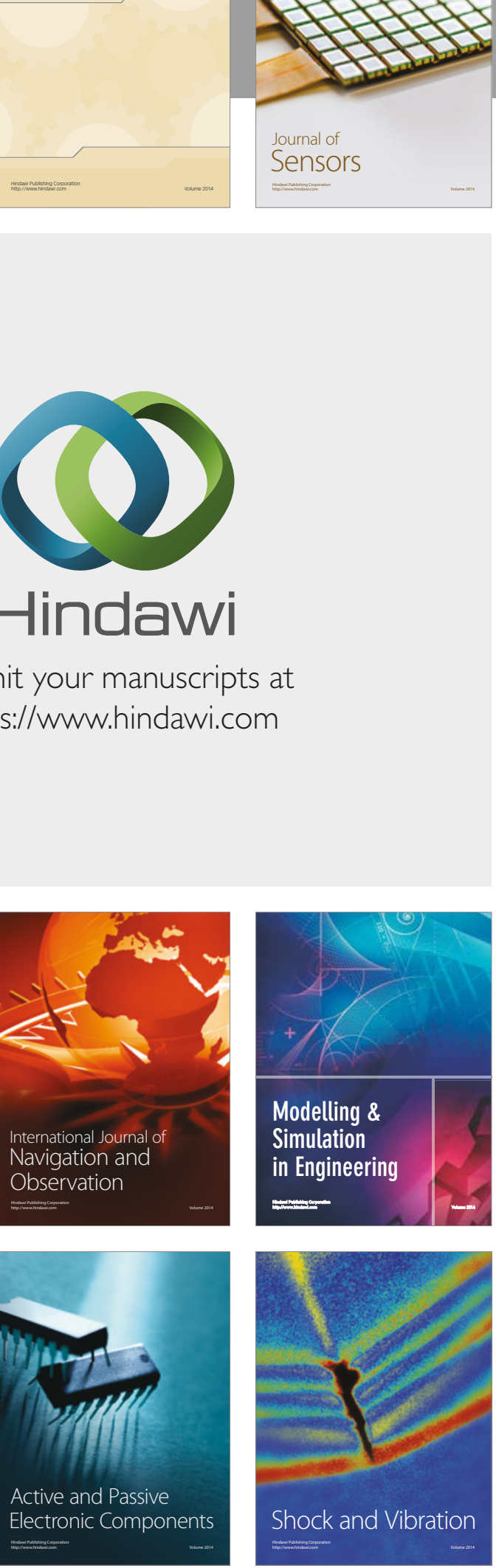
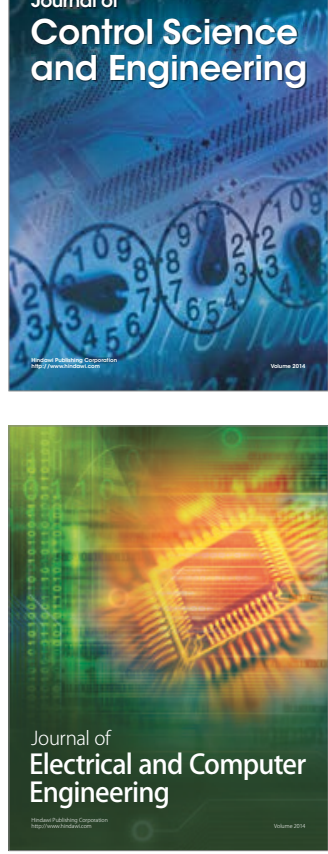

Distributed

Journal of

Control Science

and Engineering
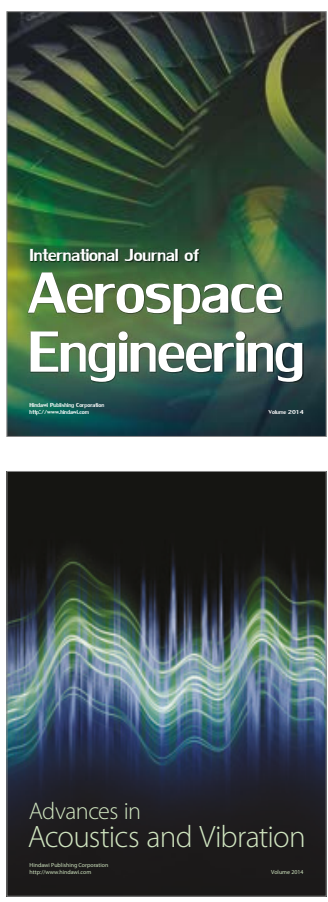

Sensor Networks 